\title{
Biorthogonal stretching of an elastic membrane beneath a uniformly rotating fluid
}

\author{
M. R. Turner \\ Department of Mathematics \\ University of Surrey \\ Guildford, Surrey, GU2 7XH \\ United Kingdom \\ Patrick D. Weidman \\ Department of Mechanical Engineering \\ University of Colorado \\ Boulder, CO 80309-0427 \\ USA
}

\begin{abstract}
The flow generated by a biorthogonally stretched membrane below a steadily rotating flow at infinity is examined. The flow's velocity field is shown to be an exact, self-similar, solution of the fully three-dimensional Navier-Stokes equations with the solution governed by a set of four ordinary differential equations. It is demonstrated that dual solutions exist when the membrane is stretched in both directions (except in the radially symmetric case), as well as for a range of parameters where the membrane is stretched in one direction and allowed to shrink in the other. For stretching rates close to the radially stretched symmetric case, four solutions exist, including one which has a large wall-jet velocity profile close to the membrane. The linear stability of each solution is also examined, and it is found that only a single solution is stable (where one exists) for a given stretching and rotation rate.
\end{abstract}

\section{Introduction}

The flow of a steadily rotating viscous fluid above an infinite flat surface has received much theoretical and experimental attention over the years. Bödewadt (1940) showed that this flow is an exact similarity solution of the three-dimensional Navier-Stokes equations, with the fluid being sucked in radially at the plate, forced upwards, and expelled at the centre of the of the rotating flow. Such exact solutions to the Navier-Stokes equations are significant because they often give insight into more complicated flows and hence identifying such flows is an active area of research interest. For example Drazin and Riley (2006), and all references therein, give a large set of exact solutions to the Navier-Stokes equations which the reader 
might find of interest.

In this paper we consider a flow similar to that studied by Bödewadt, but here the steady rotating flow occurs above an elastic membrane which can be stretched along two perpendicular axes. The case where both perpendicular stretching (or shrinking) rates are equal, i.e. a radially stretched membrane, was considered by Turner \& Weidman (2017). In their paper they showed the solution for the velocity field can again be cast as an exact similarity solution of the three-dimensional Navier-Stokes equations, and in particular that there exists a unique flow solution for each value of $a / \Omega$. Here $a$ is the membrane stretching rate and $\Omega$ is the constant angular velocity of the flow at infinity. Turner \& Weidman (2017) also examined the convective and absolute instability characteristics of these solutions and found that they were predominately unstable, except for large $a / \Omega$ values (i.e. flows where stretching dominates over rotation) where the flow stabilizes (both temporally and absolutely).

In the absence of a rotating flow at infinity, Crane (1970) investigated the two-dimensional flow induced by a stretching membrane, and found the family of exact steady solutions

$$
u(x, z)=a x e^{-\sqrt{a \nu} z}, \quad w(x, z)=\sqrt{a \nu}\left(e^{-\sqrt{a \nu} z}-1\right)
$$

where $(u, w)$ are the velocity components parallel to the usual Cartesian coordinates $(x, z)$ (with $z$ pointing perpendicular to the membrane), and $\nu$ is the kinematic viscosity of the fluid. The three-dimensional problem of a radially stretched membrane was considered by Wang (1984) who found a similar single parameter family of possible solutions, except in this case, no closed form solution was found. The case of a membrane stretched along two perpendicular axes with different stretching rates, was considered by Weidman \& Ishak (2015). There they identified dual solutions for a range of values of $\lambda=b / a$, which is the ratio of the membrane stretching rates, where one of the solutions has algebraic decay at large $z$, rather than the exponential decay observed for the second solution. In this work we revisit this problem and show that the algebraically decaying solutions are not converged solutions, and in fact we show that dual solutions only exist when the membrane is stretched along one axis, but shrunk along the other $(\lambda<0)$. For a stretched membrane along both axes $(\lambda>0)$, we show a unique solution exists.

When a biaxially stretched membrane is placed below a Hiemenz or Homann stagnation point flow, as in Weidman (2018) and Turner \& Weidman (2020) respectively, the unique set of solutions for differing stretching rates changes, and multiple solutions are found in this region. For the Hiemenz stagnation point flow, triple solutions were found in some regions of parameter spaces, while for the Homann stagnation point flow two sets of dual solutions 
were identified. In this case these branches of solutions were found to spiral together, giving an infinite set of solutions with velocity profiles which include an increasing boundary layer thickness, for the case of a membrane shrinking along both axes with different rates. In this paper we investigate the possible sets of solutions which exist when a steadily rotating flow is placed above the membrane. This work will generalize that of Weidman et al. (2017) who considered a rotating flow at infinity, above a membrane which was given a special motion which included both a shearing and stretching motion simultaneously. The problem generalization in this work allows for a pure biorthogonal stretch of the membrane to be considered.

The current paper is laid out as follows. In $\S 2$ we formulate the problem and show that the similarity solutions reduce to solving a coupled set of four ordinary differential equations, while in $\S 3$ we identify special cases of this generalized problem. In $\S 4$ we present numerical results of the governing ordinary differential equations, and the stability of these solutions is analyzed in $\S 5$. Concluding remarks are presented in $\S 6$.

\section{Problem Formulation}

We use Cartesian coordinates $(x, y, z)$ with the associated coordinate velocities $(u, v, w)$ in these directions. We assume that a elastic membrane is located at $z=0$, and the surface velocities for an impermeable membrane are

$$
u=a x, \quad v=b y, \quad w=0
$$

where $a$ is the stretching rate along the $x$-axis and $b$ is the stretching rate along $y$-axis. Here $z$ is the coordinate normal to the membrane pointing into the bulk fluid. The viscous fluid above the membrane at $z=\infty$ has uniform rotation $\Omega \mathbf{k}$ about the $z$-axis where $\Omega$ is the constant angular velocity of the flow, thus in the far field $z \rightarrow \infty$ the horizontal velocities tend to solid body rotation. For a schematic diagram of the setup, see figure 1 . The fluid density, $\rho$, and kinematic viscosity, $\nu$, are assumed to be constants. Under these conditions, the problem is governed by the equation of mass continuity

$$
u_{x}+v_{y}+w_{z}=0
$$

and the three-dimensional Navier-Stokes equations

$$
\begin{aligned}
u u_{x}+v u_{y}+w u_{z} & =-\frac{1}{\rho} p_{x}+\nu\left(u_{x x}+u_{y y}+u_{z z}\right) \\
u v_{x}+v v_{y}+w v_{z} & =-\frac{1}{\rho} p_{y}+\nu\left(v_{x x}+v_{y y}+v_{z z}\right)
\end{aligned}
$$




$$
u w_{x}+v w_{y}+w w_{z}=-\frac{1}{\rho} p_{z}+\nu\left(w_{x x}+w_{y y}+w_{z z}\right)
$$

in which $p$ is the thermodynamic pressure, and the subscripts denote partial derivatives.

We seek a solution of these equations in the form of a similarity solution where the horizontal velocity field has the ansatz

$$
u(x, y, \eta)=|a|\left(x f_{1}^{\prime}(\eta)+y f_{2}^{\prime}(\eta)\right), \quad v(y, \eta)=|a|\left(x g_{1}^{\prime}(\eta)+y g_{2}^{\prime}(\eta)\right), \quad \eta=\sqrt{\frac{|a|}{\nu} z}
$$

and the dashes denote ordinary derivatives with respect to $\eta$. Solutions of this form satisfies the continuity equation when

$$
w(\eta)=-\sqrt{\nu|a|}\left(f_{1}(\eta)+g_{2}(\eta)\right)
$$

i.e. when the axial velocity is spatially invariant in the horizontal directions. Inserting the above velocity field forms into the Navier-Stokes equations and applying the far-field conditions

$$
u(x, y, \infty)=-\Omega y, \quad v(x, y, \infty)=\Omega x
$$

yields the set of four differential equations

$$
\begin{aligned}
& f_{1}^{\prime \prime \prime}+\left(f_{1}+g_{2}\right) f_{1}^{\prime \prime}-f_{2}^{\prime} g_{1}^{\prime}-f_{1}^{2}=\sigma^{2} \\
& f_{2}^{\prime \prime \prime}+\left(f_{1}+g_{2}\right) f_{2}^{\prime \prime}-f_{2}^{\prime}\left(f_{1}^{\prime}+g_{2}^{\prime}\right)=0 \\
& g_{1}^{\prime \prime \prime}+\left(f_{1}+g_{2}\right) g_{1}^{\prime \prime}-g_{1}^{\prime}\left(f_{1}^{\prime}+g_{2}^{\prime}\right)=0 \\
& g_{2}^{\prime \prime \prime}+\left(f_{1}+g_{2}\right) g_{2}^{\prime \prime}-g_{2}^{\prime 2}-f_{2}^{\prime} g_{1}^{\prime}=\sigma^{2}
\end{aligned}
$$

in which $\sigma=\Omega /|a|$ is the dimensionless rotation parameter and $\lambda=b /|a|$ is the ratio of stretching rates. The above set of ordinary differential equations are to be solved together with boundary and far-field conditions

$$
\begin{aligned}
& f_{1}(0)=0, \quad f_{1}^{\prime}(0)= \pm 1, \quad f_{2}^{\prime}(0)=0, \quad f_{1}^{\prime}(\infty)=0, \quad f_{2}^{\prime}(\infty)=-\sigma \\
& g_{2}(0)=0, \quad g_{1}^{\prime}(0)=0, \quad g_{2}^{\prime}(0)=\lambda, \quad g_{1}^{\prime}(\infty)=\sigma, \quad g_{2}^{\prime}(\infty)=0 .
\end{aligned}
$$

Note that we have four third order ODEs with 10 boundary conditions, this is because both $f_{2}$ and $g_{1}$, introduced differentiated in (2.4), do not appear explicitly in the ODEs, and hence these can be considered as second order ODEs for $f_{2}^{\prime}$ and $g_{1}^{\prime}$ giving the correct number of boundary conditions and equations. We leave these terms differentiated in (2.4) however, for consistency and use the values $f_{2}(0)=g_{1}(0)$ without loss of generality. Also, it is clear that the $f_{2}^{\prime}$ and $g_{1}^{\prime}$ satisfy the same equations except for the sign switch in their far-field 
boundary conditions, thus $f_{2}^{\prime} \equiv-g_{1}^{\prime}$ and hence if we wish to, we need only solve for one of these quantities, or if we decide to solve for both we require this symmetry to hold. Finally we note that the governing equations do not depend upon the sign of $\sigma$, as it only appears as $\sigma^{2}$, only the sign of the boundary conditions as $\eta \rightarrow \infty$ change in (2.7a,b) if $\sigma$ changes sign. Thus we need only consider the case $\sigma>0$ with the $\sigma<0$ case being determined by switching the signs of $g_{1}(\eta)$ and $f_{2}(\eta)$.

The boundary condition $f_{1}^{\prime}(0)= \pm 1$ is to distinguish between the two cases of $a>0$ and $a<0$. In what follows a positive/negative value of $a\left(f_{1}^{\prime}(0)=+1\right.$ or $f_{1}^{\prime}(0)=-1$ in $\left.(2.7 \mathrm{a})\right)$ denotes stretching/shrinking of the membrane parallel to the $x$-axis while a positive/negative value of $b$ ( $\lambda$ positive/negative) denotes stretching/shrinking of the membrane parallel to the $y$-axis.

The system pressure field is readily found by integrating (2.3c) to be

$$
p(x, y, \eta)=p_{0}+\left(\frac{x^{2}+y^{2}}{2}\right) \rho \Omega^{2}-\rho \nu|a|\left(\frac{\left(f_{1}+g_{2}\right)^{2}}{2}+\left(f_{1}^{\prime}+g_{2}^{\prime}\right)\right)
$$

which is also independent of the rotation direction, and the wall shear stress components are given as

$$
\begin{aligned}
& \tau_{x}=\left.\mu \frac{\partial u}{\partial z}\right|_{z=0}=\rho \nu^{1 / 2}|a|^{3 / 2}\left[x f_{1}^{\prime \prime}(0)+y f_{2}^{\prime \prime}(0)\right] \\
& \tau_{y}=\left.\mu \frac{\partial v}{\partial z}\right|_{z=0}=\rho \nu^{1 / 2}|a|^{3 / 2}\left[x g_{1}^{\prime \prime}(0)+y g_{2}^{\prime \prime}(0)\right]
\end{aligned}
$$

\section{Special Cases}

The problem proposed in this paper considers general values of both $\lambda$ and $\sigma$, however, it is worth noting that the following special cases have been considered before:

- Case $\sigma=0, \lambda=0, f_{1}^{\prime}(0)=1$ : This case consists of unilateral stretching in the $x$-direction beneath quiescent fluid which was studied by Crane (1970) who found the exact solution (1.1) (i.e. $f_{1}=1-e^{-\eta}, f_{2} \equiv g_{1} \equiv g_{2} \equiv 0$ ), and the wall stress parameter given by

$$
f_{1}^{\prime \prime}(0)=-1
$$

- Case $\sigma=0, \lambda=1, f_{1}^{\prime}(0)=1$ : This case consists of a radially stretching membrane below a quiescent fluid which was studied by Wang (1984). In this case the flow was radially symmetric and so $f_{1} \equiv g_{2}$ (with $\left.f_{2} \equiv g_{1} \equiv 0\right)$ and the wall stress was found to be

$$
f_{1}^{\prime \prime}(0)=g_{2}^{\prime \prime}(0)=-1.17372
$$


- Case $\sigma=0, \lambda$ finite, $f_{1}^{\prime}(0)=1$ : This case consists of biaxial stretching below a quiescent fluid studied by Weidman \& Ishak (2015). Here $f_{2} \equiv g_{1} \equiv 0$ and dual solutions were identified for a range of $\lambda$ values.

- Case $\sigma$ finite, $\lambda=1, f_{1}^{\prime}(0)= \pm 1$ : This case comprises a radially stretching or shrinking membrane beneath a constantly rotating fluid. The problem was investigated by Turner and Weidman (2017) who found a unique similarity solution for all values of $\sigma$.

\section{Results}

In this section we present results found by numerically integrating equations (2.6) and incorporating the boundary conditions (2.7). Equations (2.6) are integrated from the membrane surface at $\eta=0$ to some large upper boundary at $\eta=\eta_{\max }$, where $\eta_{\max }$ is chosen to be large enough such that the obtained results are independent of $\eta_{\max }$. The numerical scheme used is the shooting-splitting method first presented by Firnett and Troesch (1974) which has since been utilized by the authors in related problems (Weidman and Turner, 2019; Turner and Weidman, 2020). The method splits the domain $\left[0, \eta_{\max }\right]$ into $N$ identically sized sub-domains $\left[\eta_{i}, \eta_{i+1}\right]$ for $i=0, \ldots, N$. In each of the sub-domains the vector of quantities $\mathbf{f}=\left(f_{1}, f_{1}^{\prime}, f_{1}^{\prime \prime}, f_{2}^{\prime}, f_{2}^{\prime \prime}, g_{1}^{\prime}, g_{1}^{\prime \prime}, g_{2}, g_{2}^{\prime}, g_{2}^{\prime \prime}\right)$ is integrated from $\eta_{i}$ to $\eta_{i+1}$ via a 4 th order RungeKutta method with a step size of $\Delta \eta=10^{-3}$ which we find to be small enough for results to have converged. The values of the integrated vector $\mathbf{f}$ at $\eta=\eta_{i+1}$ are then used to update the values of $\mathbf{f}$ at $\eta=\eta_{i}$ via Newton's method, by requiring that that the quantities in $\mathbf{f}$ are continuous at each $\eta_{i}$ for $i=1, \ldots, N$ and that the far-field boundary conditions are satisfied at $\eta_{N+1}$. Hence this results in a Newton iteration step where $10 N$ equations have to be solved simultaneously, and this process is continued until some convergence tolerance is met, which in this paper we set to be $\left|\mathbf{f}_{n+1}-\mathbf{f}_{n}\right|<10^{-10}$, where $n$ denotes the iteration number. For the majority of this paper we use $N=100$ sub-intervals, and set $\eta_{\max }=100$. We find this value of $\eta_{\max }$ to be significantly larger than actually needed for much of the parameter space, but as the results below will show, there are some regions of parameter space which

have velocity profiles with thick boundary layer profiles, and thus the large value of $\eta_{\max }$ is needed to deal with these values.

In the shooting-splitting method we are required to invert a $10 N \times 10 N$ Jacobian matrix, as opposed to a $4 \times 4$ Jacobian for a single-domain shooting approach, making it computationally slower and more expensive. However, this shooting-splitting approach is preferred to the single-domain approach because it is much less sensitive to the initial values of the 
unknowns, as the exponential growth of these initial 'incorrect' guesses is restricted to a short domain, hence keeping them numerically finite, and thus making it more likely that the scheme converges. This also then allows for much larger values of $\eta_{\max }$ to be considered, which we find is required to achieve converged results in this problem.

In figure $2 \mathrm{a}$ we plot the surface stress stress parameters $f_{1}^{\prime \prime}(0)$ and $g_{2}^{\prime \prime}(0)$ for the case $f_{1}^{\prime}(0)=1$ with no external flow $(\sigma=0)$, and hence the components satisfy $f_{2} \equiv g_{1} \equiv 0$. Here we see in the absence of a rotating flow that there is a single unique solution for $\lambda>0$, but there are dual solutions for $-0.251<\lambda<0$, i.e. for a stretching membrane in one direction, while shrinking in the other.

By continuing the two solutions for $-0.251<\lambda<0$ to $\lambda>0$, it may appear at first that there are in fact two solutions for $\lambda>0$ as noted in Weidman \& Ishak (2015), but it is possible to show that only one of these solutions produces a converged result in figures $2 \mathrm{~b}$ and $2 \mathrm{c}$. In figure $2 \mathrm{~b}$ we consider the two dual solutions at $\lambda=-0.1$ and plot the parameter $f_{1}^{\prime \prime}(0)$ for various values of $\eta_{\max }$. It is clear that by $\eta_{\max } \approx 40$ both solutions have converged to different results. Now fixing $\eta_{\max }=5$ and parameter continuing these results to $\lambda=1$ we still find two distinct solutions in figure $2 \mathrm{c}$, but as we increase $\eta_{\max }$ only the solid curve result converges, and in fact the dashed result appears to very slowly tend to the solid line result as $\eta_{\max }$ increases. Also on this figure are the two shear stress values identified in Weidman \& Ishak (2015) given by the dotted lines. The lower line is obscured by the solid curve as these agree exactly, while the upper line is seen not to be a converged result when compared to the dashed line, which is continued up to $\eta_{\max }=550$, which is the upper limit of what we could achieve in double precision. While this numerical result does not explicitly rule out the existence of converged results that decay algebraically as $\eta \rightarrow \infty$, we believe there to be only one unique converged result for $\sigma=0$ and $\lambda>0$. We note that using other values of $\eta_{\max }$ to parameter continue the results from $\lambda=-0.1$ leads to the same conclusion, and that on the upper $f_{1}^{\prime \prime}(0)$ branch of solutions in figure $2 \mathrm{a}$ we had to increase the value of $\eta_{\max }$ to $\eta_{\max }=500$ as we approached $\lambda=0$ from below to achieve converged results. If algebraically decaying solutions as $\eta \rightarrow \infty$ of (2.6) with $\sigma=0$ exist, then Weidman \& Ishak (2015) have shown that they have the asymptotic form $f_{1,2} \sim A_{1,2} \eta^{-1}, g_{1,2} \sim B_{1,2} \eta^{-1}$ in this limit, for constants $A_{1,2}, B_{1,2}$. Hence they can be searched for numerically, again by using the shooting-splitting method, but by including the far-field asymptotic conditions

$$
\frac{f_{1,2}^{\prime}}{f_{1,2}}=-\frac{1}{\eta_{\max }}, \quad \text { and } \quad \frac{g_{1,2}^{\prime}}{g_{1,2}}=-\frac{1}{\eta_{\max }}
$$

at $\eta=\eta_{\max }$ in the Newton update step.

In figures $3(\mathrm{a}-\mathrm{c})$ we consider the three wall stress parameters $f_{1}^{\prime \prime}(0), f_{2}^{\prime \prime}(0)$ and $g_{2}^{\prime \prime}(0)$ 
respectively, as a function of $\sigma$ for $f_{1}^{\prime}(0)=1$ and $\lambda=-0.1,0.1,0.25,0.5,0.75$ and 1 numbered 1-6 respectively. Here we see that as $\sigma$ is increased from zero the single unique solution for $\lambda>0$ (two solutions for $\lambda=-0.1$ ) becomes dual solutions for $0<\sigma<\sigma_{\max }$, except for the radially stretching case $(\lambda=1)$ which remains as a single solution for all $\sigma>0$. This result agrees with that presented in Turner and Weidman (2017). The results along the lower branch of solutions for $f_{1}^{\prime \prime}(0)$ (which corresponds to the upper branch solutions of $f_{2}^{\prime \prime}(0)$ and $\left.g_{2}^{\prime \prime}(0)\right)$ appear to have a similar behavior, i.e. $f_{1}^{\prime \prime}(0)$ decreases in magnitude away from the $\sigma=0$ value. As the value of $\lambda$ is increased, the magnitude of the difference in the wall stress values on the two branches increases greatly. In figure 4 we consider how this difference manifests itself in the forms of the velocity profiles.

Figures $4(\mathrm{a}-\mathrm{d})$ show the components of the velocity field $f_{1}^{\prime}, f_{2}^{\prime}, g_{2}^{\prime}$ and $-\left(f_{1}+g_{2}\right)$ for the results from figure 3 at $\sigma=0.2$ along the lower $f_{1}^{\prime \prime}(0)$ branch. The results show that each velocity field has a very similar structure, due mainly to the similar values of wall stress parameter values obtained. Both $f_{1}^{\prime}$ and $f_{2}^{\prime}$ have monotonically decaying boundary layer profiles from their membrane values to 0 and $-\sigma$ respectively as $\eta \rightarrow \infty$. The $g_{2}^{\prime}$ profiles are slightly different, but this is because this is the direction in which the stretching rate of the membrane is being varied. In any case, the flow in the axial direction in figure $4 \mathrm{~d}$ shows that this axial flow is always directed towards the membrane, sucking down fluid which is then ejected out parallel to the membrane at the membrane surface. This is in contrast to the zero stretching Bödewadt (1940) solution where this axial flow is directed away from the plate, suggesting that in all the presented results in figures $4(\mathrm{a}-\mathrm{d})$, the stretching of the membrane contributes the most significant component to the flow. Figures 4(e-h) show the same plots as above, except this time for solutions along the upper $f_{1}^{\prime \prime}(0)$ branch. The results for each value of $\lambda$ appear similar to the upper $f_{1}^{\prime \prime}(0)$ branch results, except when $\lambda \gtrsim 0.5$ where the velocity components parallel to the strain axes, $f_{1}^{\prime}$ and $g_{2}^{\prime}$, take on a 'wall-jet' type structure, with the maximum velocity in these directions now being located away from the membrane surface. This then sets up a strong perpendicular velocity profile $f_{2}^{\prime}$. If we now consider the axial velocity profile in figure $4 \mathrm{~h}$, we see that while for these values of $\lambda$ the axial velocity is still strictly negative (flow directed towards the membrane) there is more structure now, and the $\lambda=0.75$ result is close to changing sign near to $\eta=4$. Therefore it appears we should be able to find regions of parameter space where the axial velocity changes sign within the flow. This is significant because it creates separated regions of the flow domain because the axial velocity is spatially invariant for this similarity solution, and thus if $-\left(f_{1}+g_{2}\right)=0$ anywhere in the flow, the axial velocity is zero at this height for all $x$ and $y$.

In figure $3 \mathrm{a}$, it appears as if the solution curve for the case $\lambda=0.75$ is beginning to 
deform in such a way that it might lead to multiple solutions if $\lambda$ is increased further, and this is exactly what we find for $\lambda=0.9$ in figure 5 a. Here we observe that for $0<\sigma<1.180$ and $1.265<\sigma<1.425$ we have dual solutions, but for $1.180<\sigma<1.265$ we in fact have four possible solutions. We also note the big increase in the magnitude of the stress values on the upper $f_{1}^{\prime \prime}(0)$ branch compared to those in figure $3 \mathrm{a}$. When we consider the velocity profiles of the four different solutions at $\sigma=1.2$, labeled 1-4 in figures 5(a-e), we see that results 1 and 4 behave very similarly to the dual results in figure 4, while results 2 and 3 have behaviours which transition between the two. The most interesting result appears to be result 2 , because this result extends much further in the $\eta$ direction than the other 3 results, which have asymptoted to their far-field behaviours by $\eta \approx 30$. Result 2 does eventually asymptote to its far-field value, leading to a converged solution, but not until $\eta \approx 90$. For the axial flow in figure $5 \mathrm{e}$ we see in this case that for results 2,3 and 4 that there is a region of the flow domain where $-\left(f_{1}+g_{2}\right)>0$ and hence the axial flow is directed away from the membrane in this region (albeit a very small region for result 2). Hence for these cases the flow domain is divided into distinct regions above the membrane, between which no fluid can pass.

In figure 6 we consider the shear stress solution curves $f_{1}^{\prime \prime}(0), f_{2}^{\prime \prime}(0)$ and $g_{2}^{\prime \prime}(0)$ now as a function of $\lambda$ for the fixed values of $\sigma=0.1,0.3,0.8,1.2$ and 1.4 in panels (a)-(e) respectively. These are the equivalent $\sigma \neq 0$ plots to that in figure 2 a which depicts the $\sigma=0$ case. These figures show that the single unique solution for $\lambda>0$ with $\sigma=0$ is now a dual solution for the whole range of $\lambda$ values, except at $\lambda=1$ where the only solution is the radially symmetric result found by Turner and Weidman (2017), and the second solution asymptotes to $\lambda=1$. For $\sigma=0.1$ in figure 6 a the minimum value of $\lambda$ is given by $\lambda_{\min }=-0.208$ and so for weakly rotating flows at infinity we can still find solutions with a stretching membrane in one direction and a shrinking membrane in the orthogonal direction. When $\sigma$ is increased to $\sigma=0.3$ in figure $6 \mathrm{~b}$ this region of $\lambda<0$ solutions has almost disappeared, but the dual solutions for all $\lambda>0$ (except $\lambda=1$ ) are still observable. With the value of $\lambda_{\text {min }}$ increasing in value as $\sigma$ is increased, we wish to know what happens as this value approaches $\lambda=1$ where, from Turner and Weidman (2017), we know there is a solution for all values of $\sigma$. As $\sigma$ increases to 0.8 and 1.2 in figures $6 \mathrm{c}$ and $6 \mathrm{~d}$, we observe that the solution curves for $\lambda<1$ begin to have multiple solutions, as we saw in figure 5a. Increasing $\sigma$ further we find that that the two distinct branches coming from $\lambda=\infty$ become closer and closer, and at $\sigma \approx 1.395$ the two solutions branches touch and bifurcate. For $\sigma$ greater than this value, see $\sigma=1.4$ in figure $6 \mathrm{e}$, there is a dual set of solutions for $\lambda>1.084$ and a small region close to $\lambda=1$ where there are multiple solutions. 
In all the results presented thus far we have considered only the case when $f_{1}^{\prime}(0)=1$, i.e. when the membrane is stretched parallel to the $x$-axis. We now consider the case when $f_{1}^{\prime}(0)=-1$, i.e. the membrane is shrinking in the $x$-direction. In this case when $\lambda>0$ this is just the case of the membrane shrinking in one direction and being stretched in another, which, with a rescaling of parameters, has already been considered above. However, what hasn't been considered is whether they are any solutions with $f_{1}^{\prime}(0)=-1$ and $\lambda<0$, i.e. a shrinking membrane in both directions. From Turner and Weidman (2017) we know that the radially symmetric problem has a solution in this case, but what about the asymmetric case? In figure 7 a we consider the membrane stress parameters for the case $\sigma=0.1$ with $f_{1}^{\prime}(0)=-1$. From figure 6 a we know that there will be solution branches for $\lambda>0$ and these branches move to $\lambda=\infty$ as $\sigma$ increases, and disappear for $\sigma \gtrsim 0.3$ as found in figure $6 \mathrm{~b}$. However, for $\lambda<0$ the vertical line denotes a set of solutions close to $\lambda=-1$. The variation of this result from $\lambda=-1$ is very small, as can be seen by the blown-up image in figure $7 \mathrm{~b}$, where we plot $\lambda+1$ on the horizontal axis. We can see that the variations from $\lambda=-1$ are of $O\left(10^{-11}\right)$ for this value of $\sigma$. Increasing the value of $\sigma$ retains this set of solutions close to $\lambda=-1$ but the variation from this value reduces further, and hence we don't plot these results here.

\section{$5 \quad$ Stability of Solutions}

Having identified dual, or multiple in some cases, solutions, we now investigate the temporal stability of these solutions by considering the unsteady form of the Navier-Stokes equations (2.3). We introduce the dimensionless time variable $\tau=|a| t$ which upon inserting (2.4) and (2.5) leads to the coupled system of ordinary differential equations

$$
\begin{aligned}
& f_{1}^{\prime \prime \prime}+\left(f_{1}+g_{2}\right) f_{1}^{\prime \prime}-f_{2}^{\prime} g_{1}^{\prime}-f_{1}^{\prime 2}-f_{1 \tau}^{\prime}=\sigma^{2} \\
& f_{2}^{\prime \prime \prime}+\left(f_{1}+g_{2}\right) f_{2}^{\prime \prime}-f_{2}^{\prime}\left(f_{1}^{\prime}+g_{2}^{\prime}\right)-f_{2 \tau}^{\prime}=0 \\
& g_{1}^{\prime \prime \prime}+\left(f_{1}+g_{2}\right) g_{1}^{\prime \prime}-g_{1}^{\prime}\left(f_{1}^{\prime}+g_{2}^{\prime}\right)-g_{1 \tau}^{\prime}=0 \\
& g_{2}^{\prime \prime \prime}+\left(f_{1}+g_{2}\right) g_{2}^{\prime \prime}-g_{2}^{\prime 2}-f_{2}^{\prime} g_{1}^{\prime}-g_{2 \tau}^{\prime}=\sigma^{2} .
\end{aligned}
$$

To study the temporal stability of these equations we follow the approach laid out in works such as Turner and Weidman (2019;2020). We split the flow into a steady basic flow element, and a small amplitude, time-dependent perturbation in the form

$$
\left[f_{1}, f_{2}, g_{1}, g_{2}\right](\eta, \tau)=\left[f_{10}, f_{20}, g_{10}, g_{20}\right](\eta)+\delta e^{-\kappa \tau}\left[F_{1}, F_{2}, G_{1}, G_{2}\right](\eta)
$$


where $\delta \ll 1$, and $\kappa$ is an eigenvalue, where $\kappa<0$ denotes an unstable solution. The quantities $f_{10}, f_{20}, g_{10}$ and $g_{20}$ are the basic flow solutions of (2.6) found in $\S 4$, while at $O(\delta)$ the perturbation quantities satisfy the linear system

$$
\begin{gathered}
F_{1}^{\prime \prime \prime}+\left(f_{10}+g_{20}\right) F_{1}^{\prime \prime}+f_{10}^{\prime \prime}\left(F_{1}+G_{2}\right)-f_{20}^{\prime} G_{1}^{\prime}-g_{10}^{\prime} F_{2}^{\prime}-2 f_{10}^{\prime} F_{1}^{\prime}+\kappa F_{1}^{\prime}=0 \\
F_{2}^{\prime \prime \prime}+\left(f_{10}+g_{20}\right) F_{2}^{\prime \prime}+f_{20}^{\prime \prime}\left(F_{1}+G_{2}\right)-f_{20}^{\prime}\left(F_{1}^{\prime}+G_{2}^{\prime}\right)-\left(f_{10}^{\prime}+g_{20}^{\prime}\right) F_{2}^{\prime}+\kappa F_{2}^{\prime}=0 \\
G_{1}^{\prime \prime \prime}+\left(f_{10}+g_{20}\right) G_{1}^{\prime \prime}+g_{10}^{\prime \prime}\left(F_{1}+G_{2}\right)-g_{10}^{\prime}\left(F_{1}^{\prime}+G_{2}^{\prime}\right)-\left(f_{10}^{\prime}+g_{20}^{\prime}\right) G_{1}^{\prime}+\kappa G_{1}^{\prime}=0 \\
G_{2}^{\prime \prime \prime}+\left(f_{10}+g_{20}\right) G_{2}^{\prime \prime}+g_{10}^{\prime \prime}\left(F_{1}+G_{2}\right)-2 g_{20}^{\prime} G_{2}^{\prime}-f_{20}^{\prime} G_{1}^{\prime}-g_{10}^{\prime} F_{2}^{\prime}+\kappa G_{2}^{\prime}=0 .
\end{gathered}
$$

The above system is then solved with the homogeneous boundary conditions

$$
\begin{gathered}
F_{1}(0)=F_{1}^{\prime}(0)=F_{2}^{\prime}(0)=F_{1}^{\prime}(\infty)=F_{2}^{\prime}(\infty)=0 \\
G_{2}(0)=G_{1}^{\prime}(0)=G_{2}^{\prime}(0)=G_{1}^{\prime}(\infty)=G_{2}^{\prime}(\infty)=0
\end{gathered}
$$

using the same numerical scheme as for the base flow. We are again free to choose the extra conditions $F_{2}(0)=G_{1}(0)=0$ as these functions do not appear explicitly in (5.3), and we fix $F_{1}^{\prime \prime}(0)=1$. This leaves four unknown variables to determine in order to fully solve the system, namely $F_{2}^{\prime \prime}(0), G_{1}^{\prime \prime}(0), G_{2}^{\prime \prime}(0)$ and $\kappa$, which are updated via Newton iterations in order to satisfy the far-field boundary conditions (5.4). Results of the form (5.2) produce an infinite set of real eigenvalues $\kappa_{1}<\kappa_{2}<\kappa_{3}<\cdots$, where our interest lies in determining the value of $\kappa_{1}$. If $\kappa_{1}>0$ then the resulting flow is stable and we would expect to observe it in an experiment, while if $\kappa_{1}<0$ then the flow is unstable and we would not expect to observe it.

The calculation of the eigenvalues for these stretching plate flows is tricky, because as was shown by Davies and Pozrikidis (2014) for the two-dimensional Crane flow (1.1), the perturbation eigenmodes are able to penetrate a large distance into the main bulk of the fluid due to the weak convection towards the membrane in the far-field, given by $f_{10}(\infty)+g_{20}(\infty)=$ $w_{\infty}$. The same is true for the problem considered here, and in the limit as $\eta \rightarrow \infty$ (5.3) can be written as

$$
\begin{aligned}
& F_{1}^{\prime \prime \prime}+w_{\infty} F_{1}^{\prime \prime}+\sigma G_{1}^{\prime}-\sigma F_{2}^{\prime}+\kappa F_{1}^{\prime}=0 \\
& F_{2}^{\prime \prime \prime}+w_{\infty} F_{2}^{\prime \prime}+\sigma\left(F_{1}^{\prime}+G_{2}^{\prime}\right)+\kappa F_{2}^{\prime}=0 \\
& G_{1}^{\prime \prime \prime}+w_{\infty} G_{1}^{\prime \prime}-\sigma\left(F_{1}^{\prime}+G_{2}^{\prime}\right)+\kappa G_{1}^{\prime}=0 \\
& G_{2}^{\prime \prime \prime}+w_{\infty} G_{2}^{\prime \prime}+\sigma G_{1}^{\prime}-\sigma F_{2}^{\prime}+\kappa G_{2}^{\prime}=0 .
\end{aligned}
$$


This constant coefficient system can be solved by seeking exponential solutions of the form

$$
\left[F_{1}, F_{2}, G_{1}, G_{2}\right]=[A, B, C, D] e^{q \eta}
$$

where $A, B, C$ and $D$ are constants, leading to the matrix problem

$$
\left[\begin{array}{cccc}
q\left(q^{2}+w_{\infty} q+\kappa\right) & -\sigma q & \sigma q & 0 \\
\sigma q & q\left(q^{2}+w_{\infty} q+\kappa\right) & 0 & \sigma q \\
-\sigma q & 0 & q\left(q^{2}+w_{\infty} q+\kappa\right) & -\sigma q \\
0 & -\sigma q & \sigma q & q\left(q^{2}+w_{\infty} q+\kappa\right)
\end{array}\right]\left[\begin{array}{c}
A \\
B \\
C \\
D
\end{array}\right]=0
$$

Nontrivial solutions to this system lead to seven independent values of the eigenmode decay rate $q$

$$
\begin{gathered}
q_{1}=0, \quad q_{2}=-\frac{w_{\infty}}{2}+\sqrt{\frac{w_{\infty}^{2}}{4}-\kappa}, \quad q_{3}=-\frac{w_{\infty}}{2}-\sqrt{\frac{w_{\infty}^{2}}{4}-\kappa}, \\
q_{4}=-\frac{w_{\infty}}{2}+\sqrt{\frac{w_{\infty}^{2}}{4}-\kappa-2 \mathrm{i} \sigma}, \quad q_{5}=-\frac{w_{\infty}}{2}-\sqrt{\frac{w_{\infty}^{2}}{4}-\kappa-2 \mathrm{i} \sigma} \\
q_{6}=-\frac{w_{\infty}}{2}+\sqrt{\frac{w_{\infty}^{2}}{4}-\kappa+2 \mathrm{i} \sigma}, \quad q_{7}=-\frac{w_{\infty}}{2}-\sqrt{\frac{w_{\infty}^{2}}{4}-\kappa+2 \mathrm{i} \sigma .}
\end{gathered}
$$

In our extensive search of parameter space, we only identified real values of $\kappa$, in which case $q_{6}=\overline{q_{4}}$ and $q_{7}=\overline{q_{5}}$ where $\overline{(\cdot)}$ denotes the complex conjugate, and $q_{2}$ and $q_{3}$ are complex conjugates for $\kappa>w_{\infty}^{2} / 4$. In $\S 4$ we found that $w_{\infty}>0$ but this value is a function of $\lambda$ and $\sigma$, and so the expected exponential decay of the eigenmode is hard to predict, making the numerical calculations tricky due to the eigenvalue being relatively dependent on the domain truncation size $\eta_{\max }$. However, we find the shooting-splitting method with $\eta_{\max }=100$ suitable to handle this problem and gives domain independent results.

Figure $8 \mathrm{a}$ plots the value of $\kappa_{1}$ for the $\sigma=0$ result from figure 2a, and shows that for $\lambda>0$, where there is a unique solution, this solution is stable, and in fact the stability of the solution increases with increasing $\lambda$. At $\lambda=0$ the growth rate is $\kappa_{1}=\frac{1}{4}$ in agreement with the result of Davis and Pozrikidis (2014) and for $\lambda<0$, one of the dual solutions is stable (the lower $f_{1}^{\prime \prime}(0)$ branch from figure $2 \mathrm{a}$ ) and one is unstable (the upper $f_{1}^{\prime \prime}(0)$ branch from figure 2a), with the change in behavior occurring at the turning point $\lambda_{\min }=-0.251$. In figure $8 \mathrm{~b}$ we examine the stability of the dual solutions from figure 3 with $\sigma \neq 0$, in particular we plot $\kappa_{1}$ for $\lambda=-0.1,0.5$ and 1 . We find that for both $\lambda=-0.1$ and 0.5 only the lower $f_{1}^{\prime \prime}(0)$ branch solution in figure $3 \mathrm{a}$ is stable, with the turning point denoting the change in stability. For the $\lambda=1$ case there is no lower $f_{1}^{\prime \prime}(0)$ branch, and we find that $\kappa_{1} \rightarrow 0$ from above as $\sigma$ is increased, and for $\sigma \gtrsim 1.395$, the value of $\kappa_{1}<10^{-3}$, thus the solutions are approximately neutrally stable beyond this point. This value of $\sigma \approx 1.395$ is 
the same value of $\sigma$ where we found the two solutions branches touch and bifurcate in figure 6. In terms of the stability of the branches of solutions plotted in figure 6 , this means that only the $f_{1}^{\prime \prime}(0)$ branch of solutions which connects the turning point $\lambda_{\min }$ to $\lambda=\infty$ is stable (see figures $6 \mathrm{a}, 6 \mathrm{~b}, 6 \mathrm{c}$ and $6 \mathrm{~d}$ ), while after the pinching of the branch solutions has occurred (see figure 6e) then only the upper $f_{1}^{\prime \prime}(0)$ branch of solutions connecting $\lambda_{\min }$ and $\lambda=\infty$ is stable. The stability results in figure $8 \mathrm{~b}$ then suggests that the symmetric solution at $\lambda=1$ is approximately neutrally stable, and in fact we find moving away from this solution leads to an unstable solution.

\section{Conclusions}

In this paper we examined the flow generated by a biorthogonally stretched membrane below a steadily rotating fluid. The problem was non-dimensionalized such that the stretching rates of the membrane along the orthogonal axes were 1 (or -1 ) and $\lambda$ respectively, while the rotation rate at $\eta=\infty$ was $\sigma$, where $\eta$ is a non-dimensional coordinate measured perpendicular to the membrane. Note that a negative stretching rate corresponds to a steadily shrinking membrane. The results showed that for a fixed value of $\lambda>\lambda_{\text {min }}$ there are two solution branches in the $\left(\sigma, f_{1}^{\prime \prime}(0)\right)$-plane where $f_{1}^{\prime \prime}(0)$ is proportional to the shear stress at the membrane along one of the stretching axes. No solutions exist for $\lambda<\lambda_{\text {min }}$. The results also showed that for each $\lambda$ there was a maximum value of $\sigma=\sigma_{\max }$ above which it was not possible to find solutions of the similarity type sought here.

For a fixed value of $\sigma$ and $\lambda$ it is shown that only one solution branch is stable to threedimensional perturbations, while the remaining branch is unstable. Along the stable branch the velocity profiles parallel to the surface of the membrane mainly have a boundary layer type profile where the maximum flow value lies at the membrane itself. As $\lambda$ is increased, the thickness of the boundary layer thins, which is accompanied by an increased axial flow towards the membrane from infinity. Along the unstable branch the velocity profiles have a 'wall-jet' type structure with the maximum flow velocity now located away from the wall in the bulk of the fluid.

\section{References}

Bödewadt, U. T., Die Dreshstömung über festem Grund, Z. Angew Math. Mech., bf 20 (1940) 241-253. 
Crane, L. J., Flow past a stretching plate, ZAMP, 21 (1970) 645-647.

Davis, J. M. and Pozrikidis, C., Linear stability of viscous flow induced by surface stretching. Arch. Appl. Mech, 84 (2014) 985-998.

Drazin, P. and Riley, N., The Navier-Stokes Equations: A Classification of Flows and Exact Solutions, London Mathematical Society Lecture Note Series 334 (Cambridge University Press, 2006).

Firnett, P. J. and Troesch, B. A., Shooting-splitting method for sensitive two-point boundary value problems, In: Bettis D.G. (eds) Proceedings of the Conference on the Numerical Solution of Ordinary Differential Equations, 362, 408-433 (Springer-Verlag, Berlin, 1974).

Turner, M. R. and Weidman, P. D., Stability of a radially stretching disk beneath a uniformly rotating fluid. Phys. Rev. Fluids, 2 (2017) 073904.

Turner, M. R. and Weidman, P. D., Impinging Howarth stagnation-point flows. Euro. J. Mech. B/Fluids, 74 (2019) 242-251.

Turner, M. R. and Weidman, P. D., Homann stagnation-point flow impinging on a biaxially stretching surface. Euro. J. Mech. B/Fluids, 86 (2020) 49-56.

Wang, C. Y., The three-dimensional flow due to a stretching flat surface, Phys. Fluids, 27 (1984) 1915-1917.

Weidman, P. D., Hiemenz stagnation-point flow impinging on a biaxially stretching surface. Meccanica, 53(4) (2018) 833-840.

Weidman, P. D. and Ishak, A., Multiple solutions of two-dimensional and three-dimensional flows induced by a stretching flat surface. Commun. Nonlinear Sci. Numer. Simul., 25 (2015) 1-9.

Weidman, P. D., Mansur, S. and Ishak, A., Biorthogonal stretching and shearing of an impermeable surface in a uniformly rotating fluid system. Meccanica, 52 (2017) 1515-1525.

Weidman, P. D. and Turner, M. R., The steady flow of one uniformly rotating fluid layer above another immiscible uniformly rotating fluid layer. Phys. Rev. Fluids, 4 (2019) 084002. 


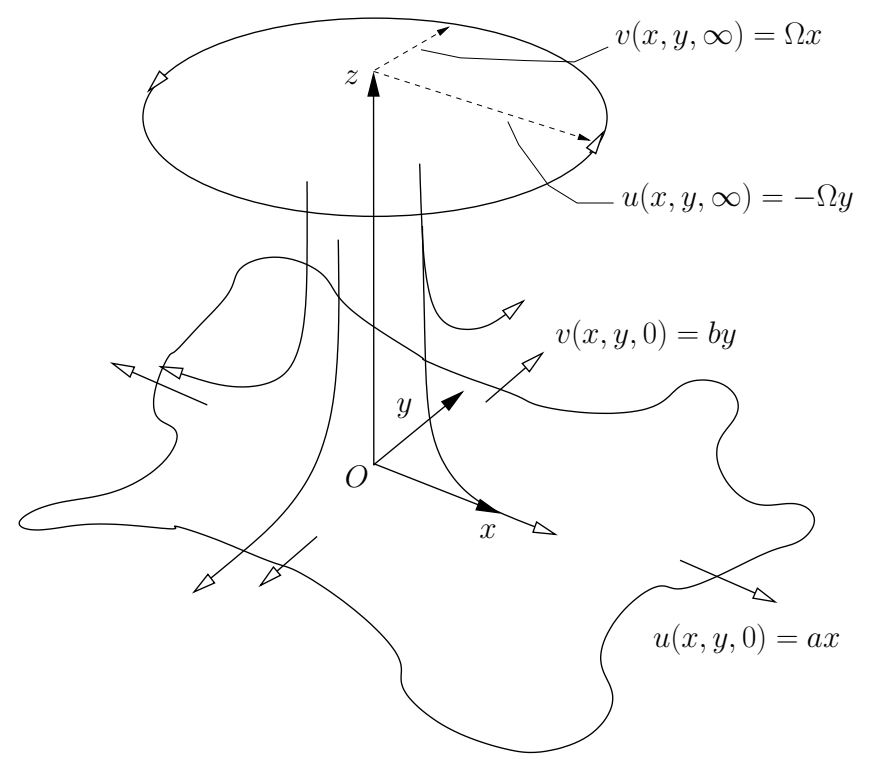

Figure 1. Schematic diagram of an orthogonally stretched plate in twodimensions below a constantly rotating flow with angular velocity $\Omega$ at $z=\infty$.

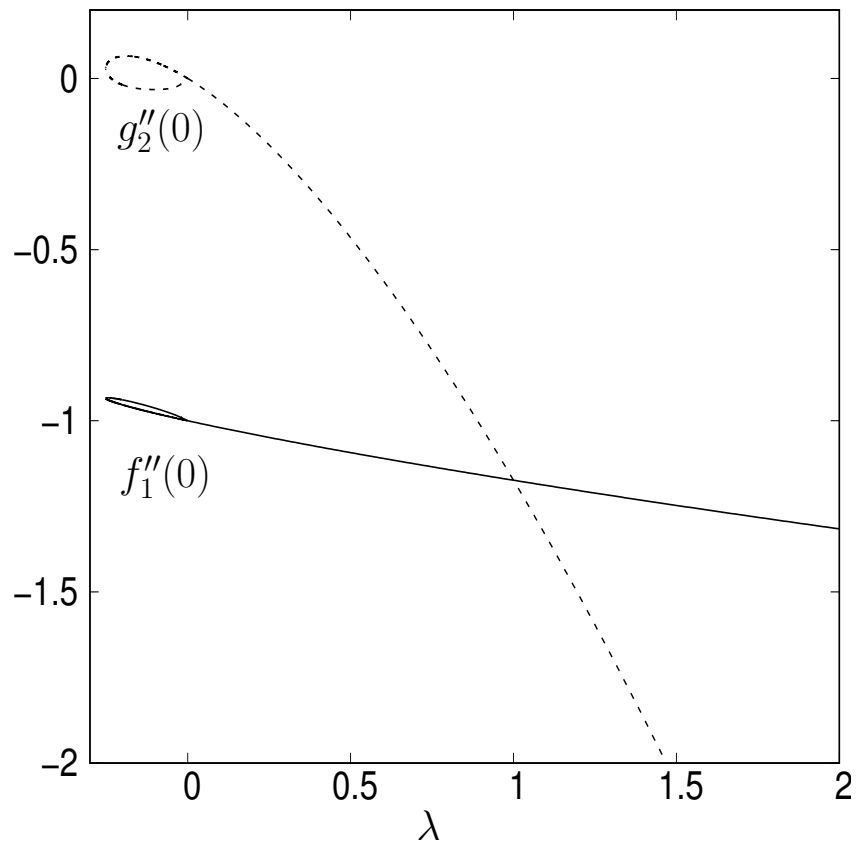

Figure 2a. Plate stress parameters $f_{1}^{\prime \prime}(0)$ (solid curve) and $g_{2}^{\prime \prime}(0)$ (dashed curve) as a function of $\lambda$ for $\sigma=0$. The turning points occur at $\left(\lambda_{\min }, f_{1}^{\prime \prime}(0), g_{2}^{\prime \prime}(0)\right)=$ $(-0.251,-0.935,0.031)$. Note there is a unique solution for $\lambda \geq 0$ and dual solutions for $\lambda_{\min }<\lambda<0$. 


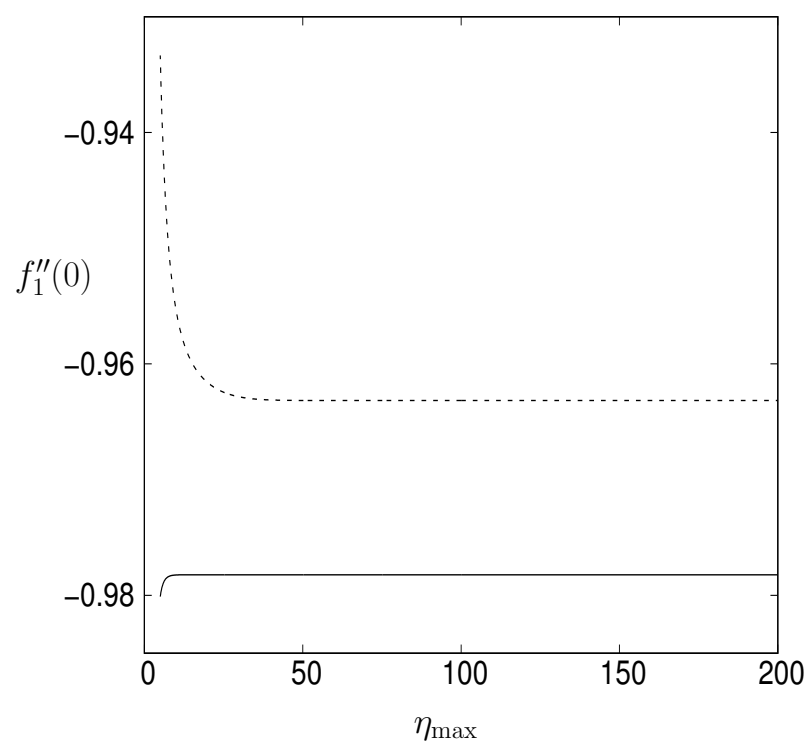

Figure 2b. Plate stress parameter $f_{1}^{\prime \prime}(0)$ as a function of $\eta_{\max }$ for $\lambda=-0.1$ and $\sigma=0$. The lower $f_{1}^{\prime \prime}(0)$ branch solution is given by the solid curve and the upper branch solution is given by the dashed curve.

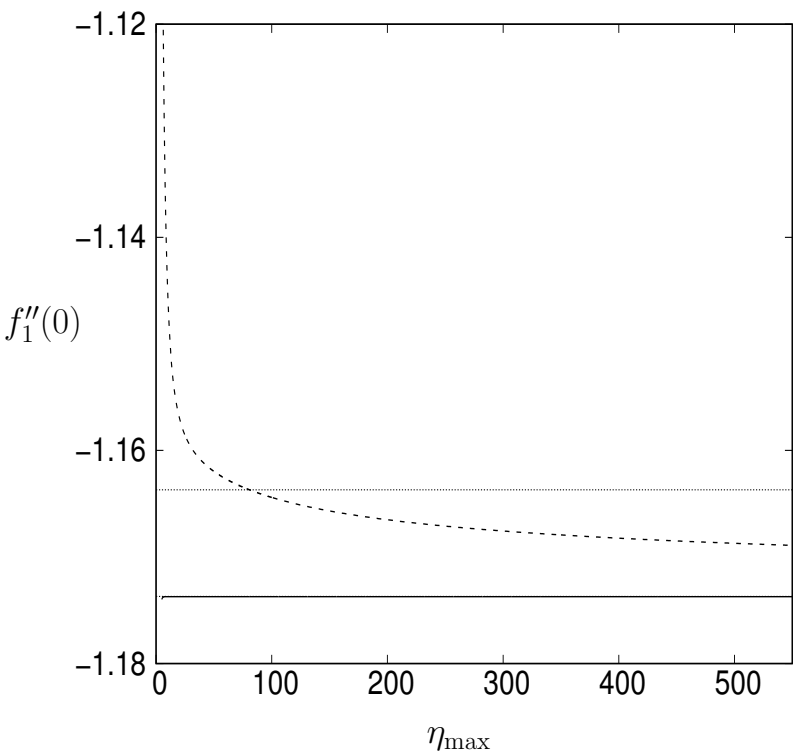

Figure 2c. Plate stress parameter $f_{1}^{\prime \prime}(0)$ as a function of $\eta_{\max }$ for $\lambda=1$ and $\sigma=0$. The solid curve is the lower $f_{1}^{\prime \prime}(0)$ branch solution from figure $2 \mathrm{~b}$ parameter continued from $\lambda=-0.1$ with $\eta_{\max }=5$ while the dashed curve is the upper $f_{1}^{\prime \prime}(0)$ branch solution from figure $2 \mathrm{~b}$ parameter continued from $\lambda=-0.1$ with $\eta_{\max }=5$. Only the lower branch solution definitely converges for the values of $\eta_{\max }$ calculated. The two dotted lines give the 'converged' results for the two branches quoted in Weidman \& Ishak (2015). 


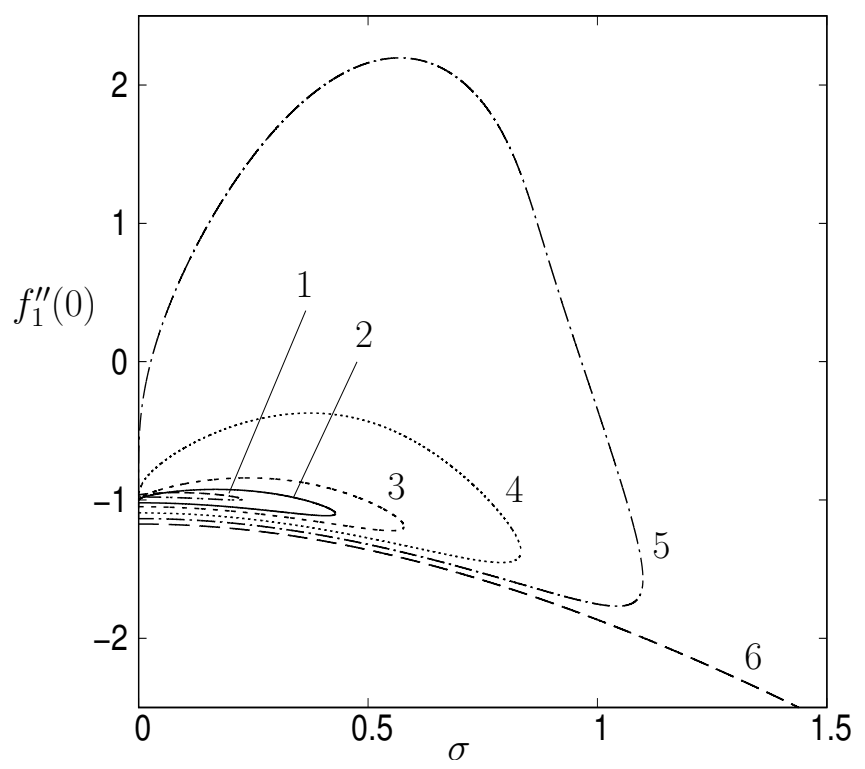

Figure 3a. Plate stress parameter $f_{1}^{\prime \prime}(0)$ as a function of $\sigma$ for $\lambda=$ $-0.1,0.1,0.25,0.5,0.75$ and 1.0 labeled $1-6$. The maximum values $\sigma_{\max }$ for the results shown are $0.226,0.429,0.579,0.834,1.099$ and $\infty$ respectively.

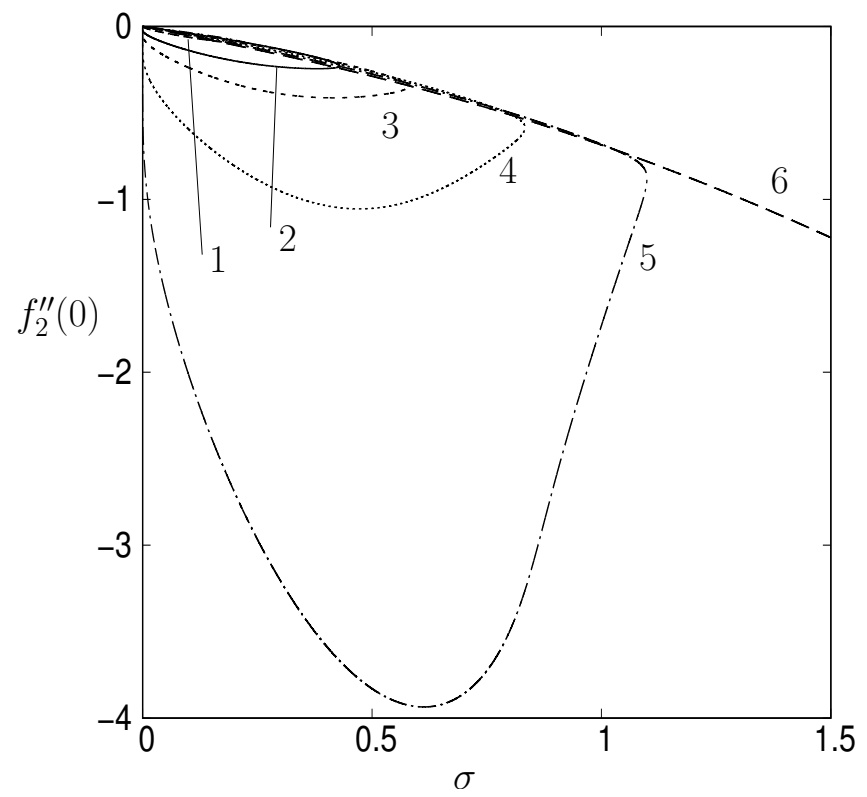

Figure 3b. Plate stress parameter $f_{2}^{\prime \prime}(0)$ as a function of $\sigma$ for $\lambda=$ $-0.1,0.1,0.25,0.5,0.75$ and 1.0 labeled $1-6$. The maximum values $\sigma_{\max }$ for the results shown are $0.226,0.429,0.579,0.834,1.099$ and $\infty$ respectively. 


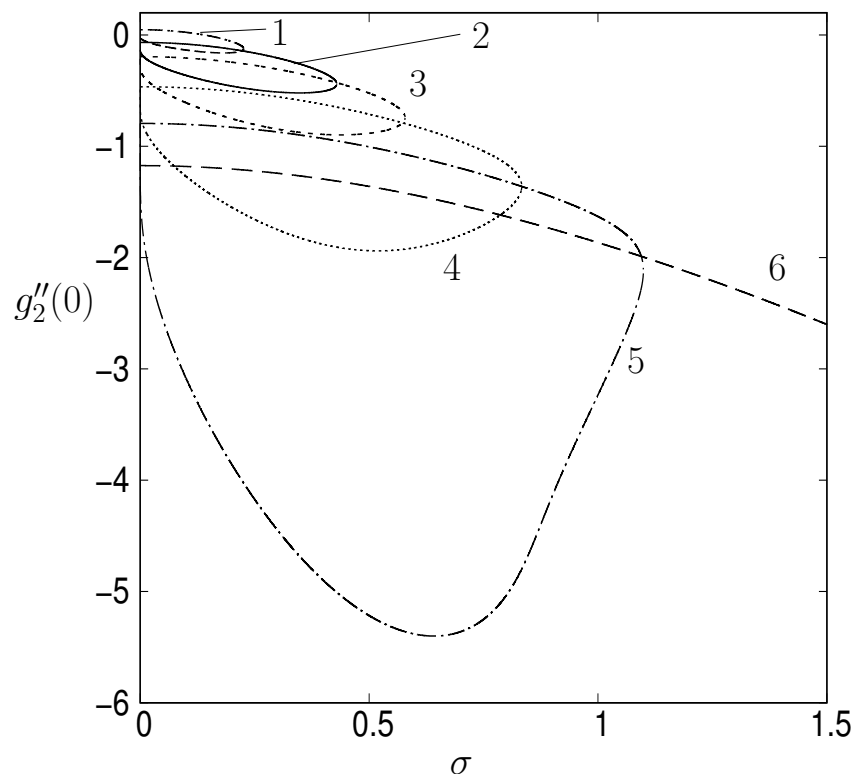

Figure 3c. Plate stress parameter $g_{2}^{\prime \prime}(0)$ as a function of $\sigma$ for $\lambda=$ $-0.1,0.1,0.25,0.5,0.75$ and 1.0 labeled 1-6. The maximum values $\sigma_{\max }$ for the results shown are $0.226,0.429,0.579,0.834,1.099$ and $\infty$ respectively.

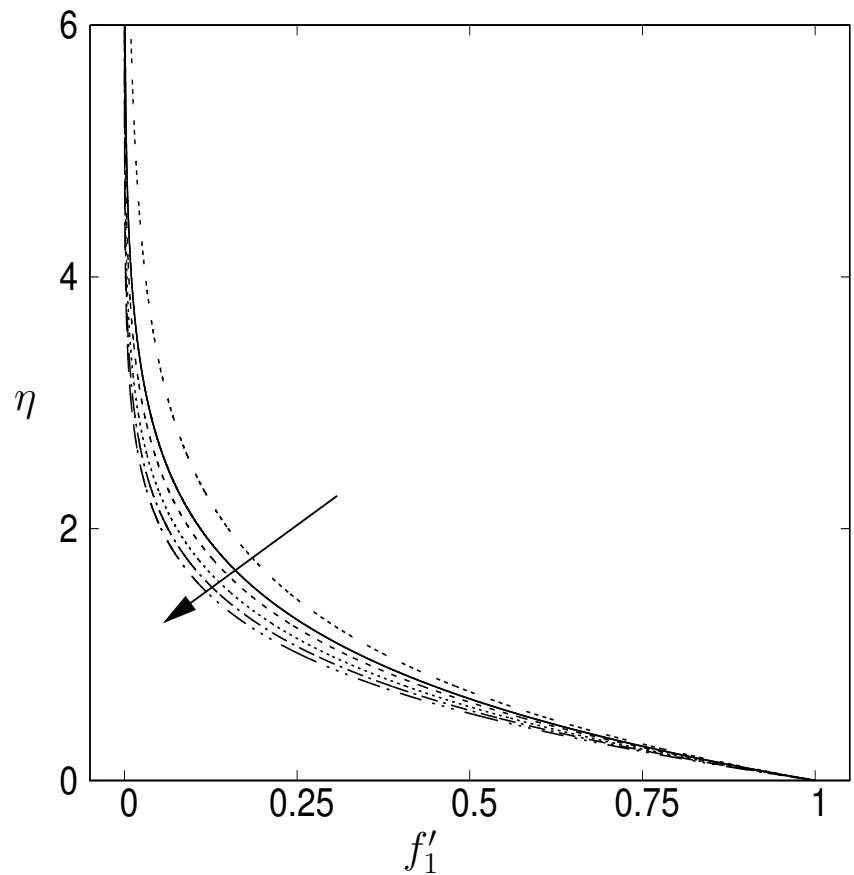

Figure 4a. Velocity profile $f_{1}^{\prime}(\eta)$ for $\sigma=0.2$ and $\lambda=-0.1,0.1,0.25,0.5,0.75$ and 1 along the lower $f_{1}^{\prime \prime}(0)$ branch. The arrow indicates the direction of increasing $\lambda$. 


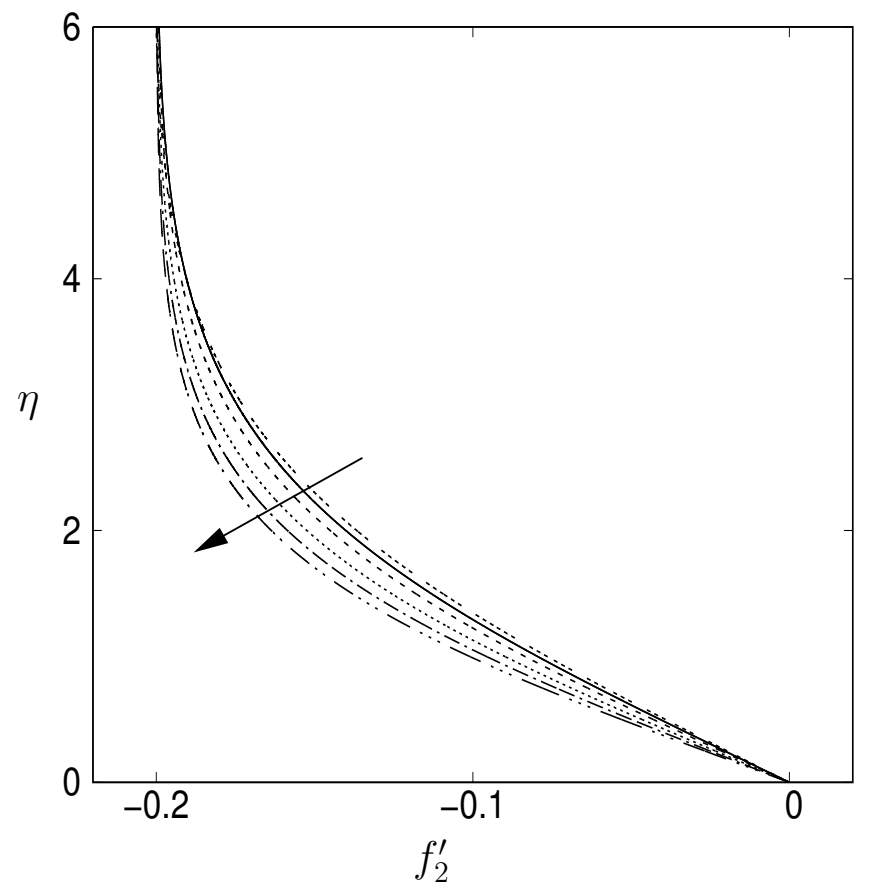

Figure 4 b. Velocity profile $f_{2}^{\prime}(\eta)$ for $\sigma=0.2$ and $\lambda=-0.1,0.1,0.25,0.5,0.75$ and 1 along the lower $f_{1}^{\prime \prime}(0)$ branch. The arrow indicates the direction of increasing $\lambda$.

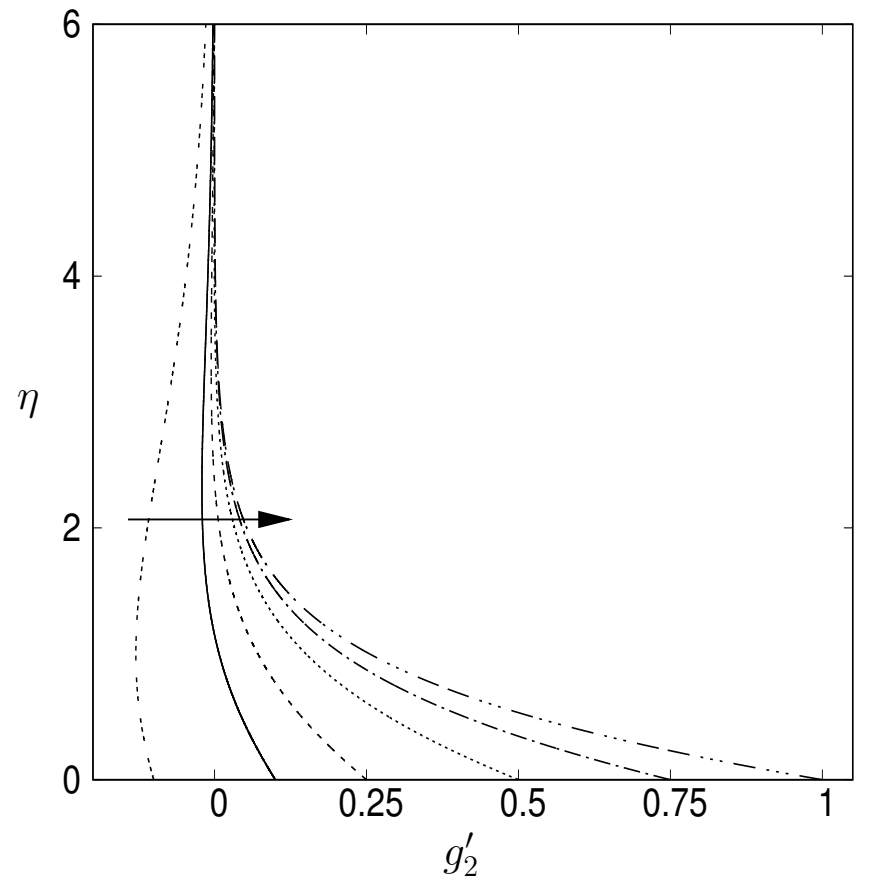

Figure 4c. Velocity profile $g_{2}^{\prime}(\eta)$ for $\sigma=0.2$ and $\lambda=-0.1,0.1,0.25,0.5,0.75$ and 1 along the lower $f_{1}^{\prime \prime}(0)$ branch. The arrow indicates the direction of increasing $\lambda$. 


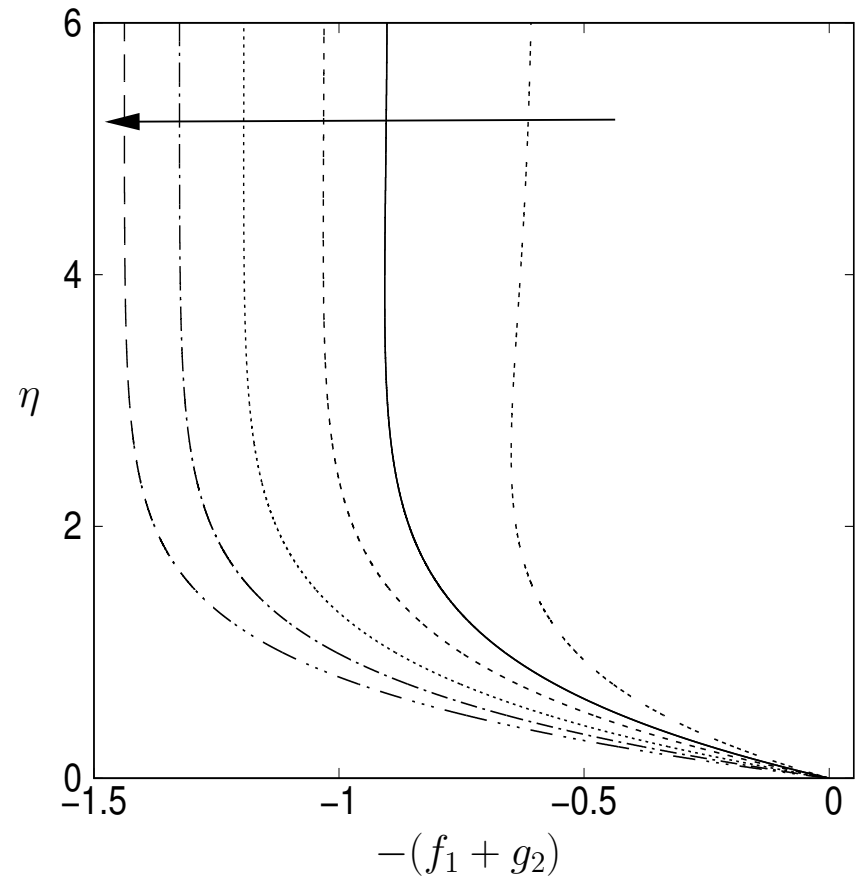

Figure 4d. Velocity profile $-\left(f_{1}+g_{2}\right)(\eta)$ for $\sigma=0.2$ and $\lambda=$ $-0.1,0.1,0.25,0.5,0.75$ and 1 along the lower $f_{1}^{\prime \prime}(0)$ branch. The arrow indicates the direction of increasing $\lambda$.

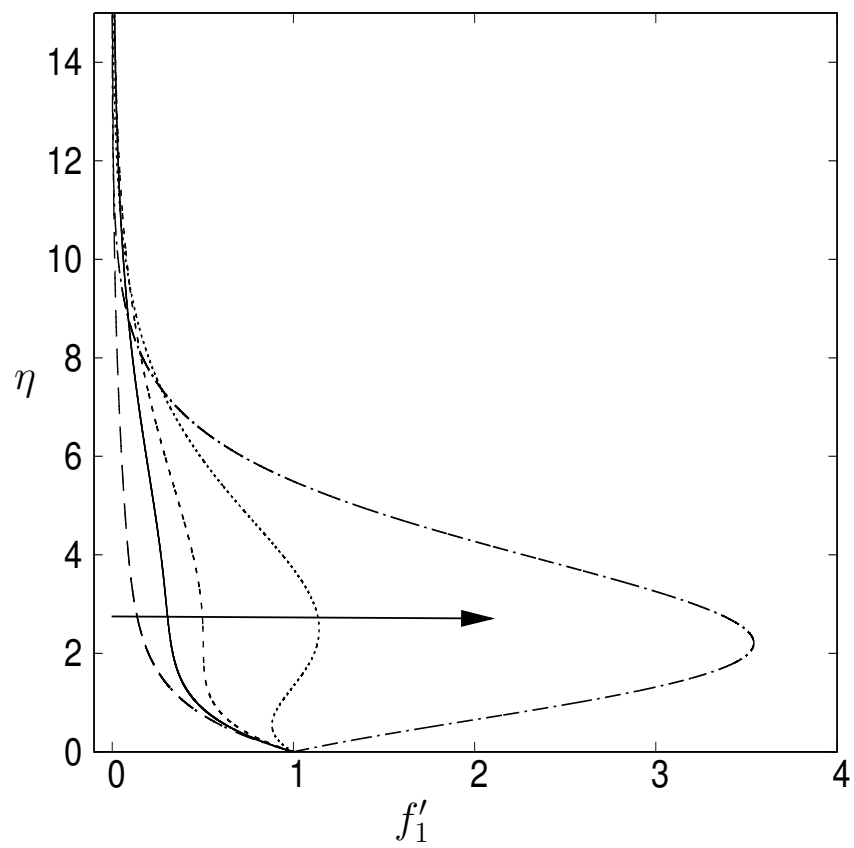

Figure 4e. Velocity profile $f_{1}^{\prime}(\eta)$ for $\sigma=0.2$ and $\lambda=-0.1,0.1,0.25,0.5$ and 0.75 along the upper $f_{1}^{\prime \prime}(0)$ branch. The arrow indicates the direction of increasing $\lambda$. 


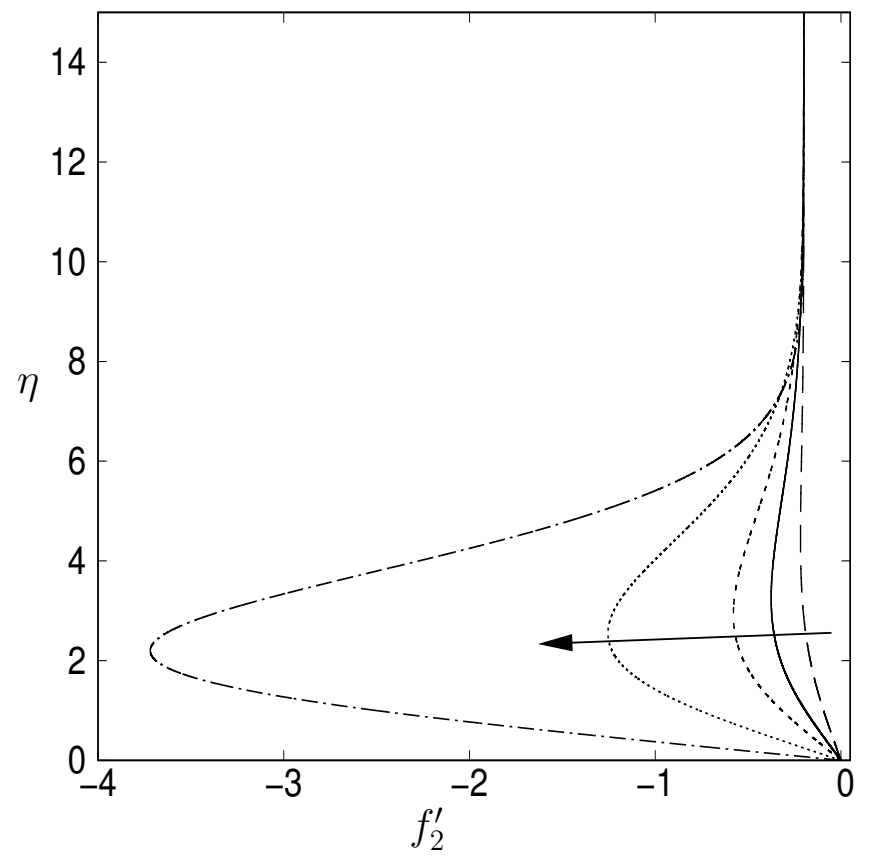

Figure 4f. Velocity profile $f_{2}^{\prime}(\eta)$ for $\sigma=0.2$ and $\lambda=-0.1,0.1,0.25,0.5$ and 0.75 along the upper $f_{1}^{\prime \prime}(0)$ branch. The arrow indicates the direction of increasing $\lambda$.

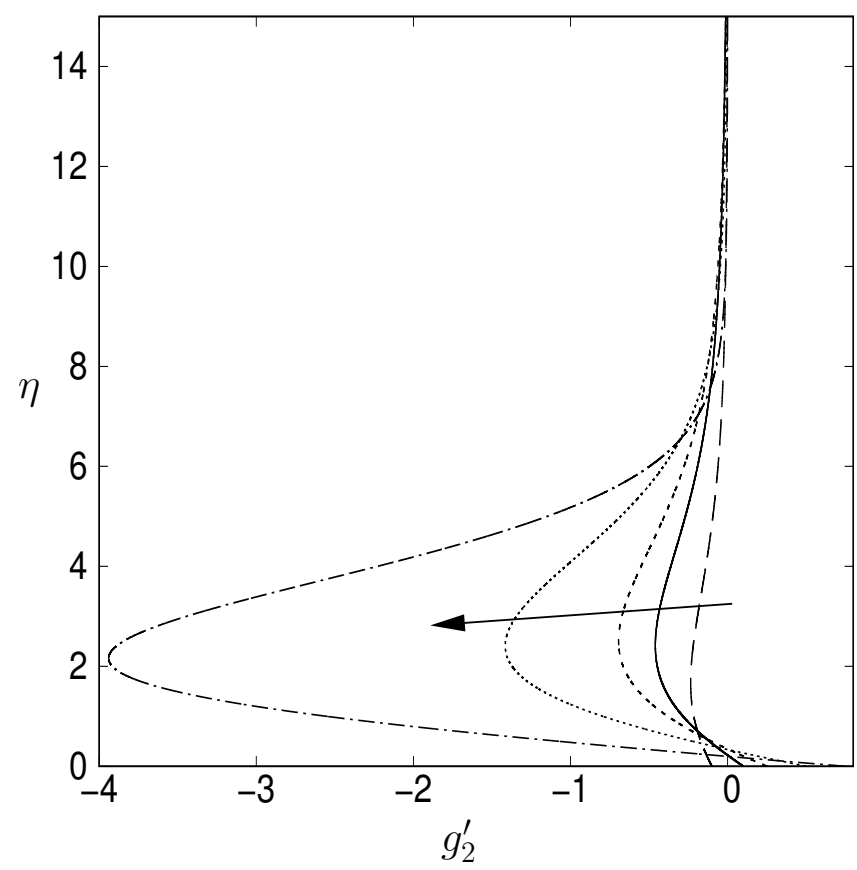

Figure 4g. Velocity profile $g_{2}^{\prime}(\eta)$ for $\sigma=0.2$ and $\lambda=-0.1,0.1,0.25,0.5$ and 0.75 along the upper $f_{1}^{\prime \prime}(0)$ branch. The arrow indicates the direction of increasing $\lambda$. 


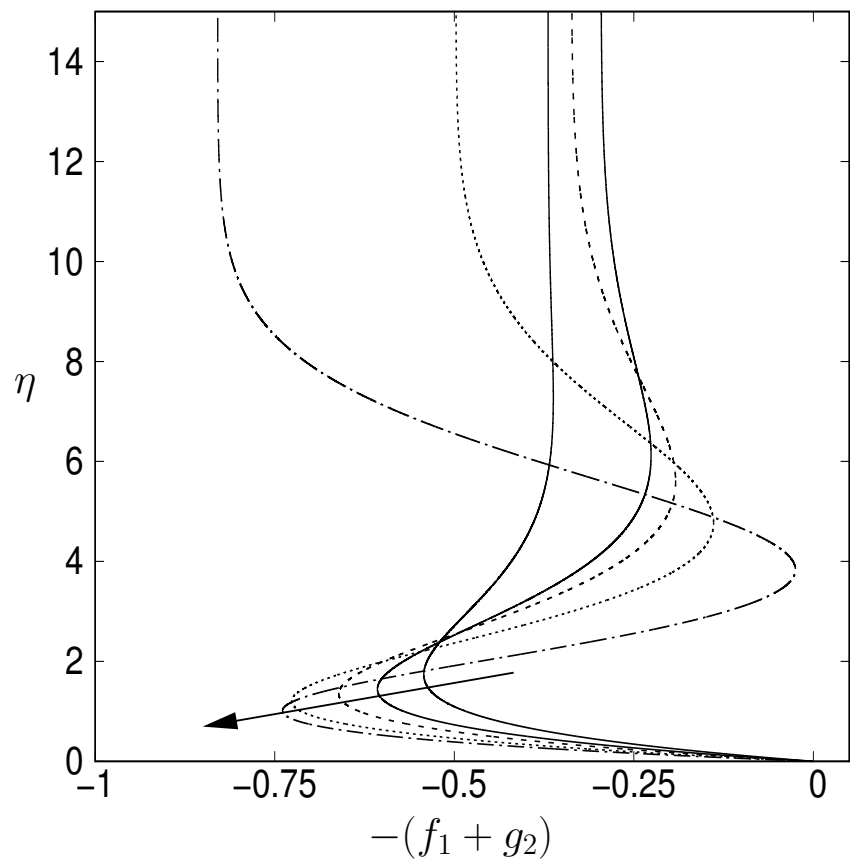

Figure 4h. Velocity profile $-\left(f_{1}+g_{2}\right)(\eta)$ for $\sigma=0.2$ and $\lambda=-0.1,0.1,0.25,0.5$ and 0.75 along the upper $f_{1}^{\prime \prime}(0)$ branch. The arrow indicates the direction of increasing $\lambda$. 


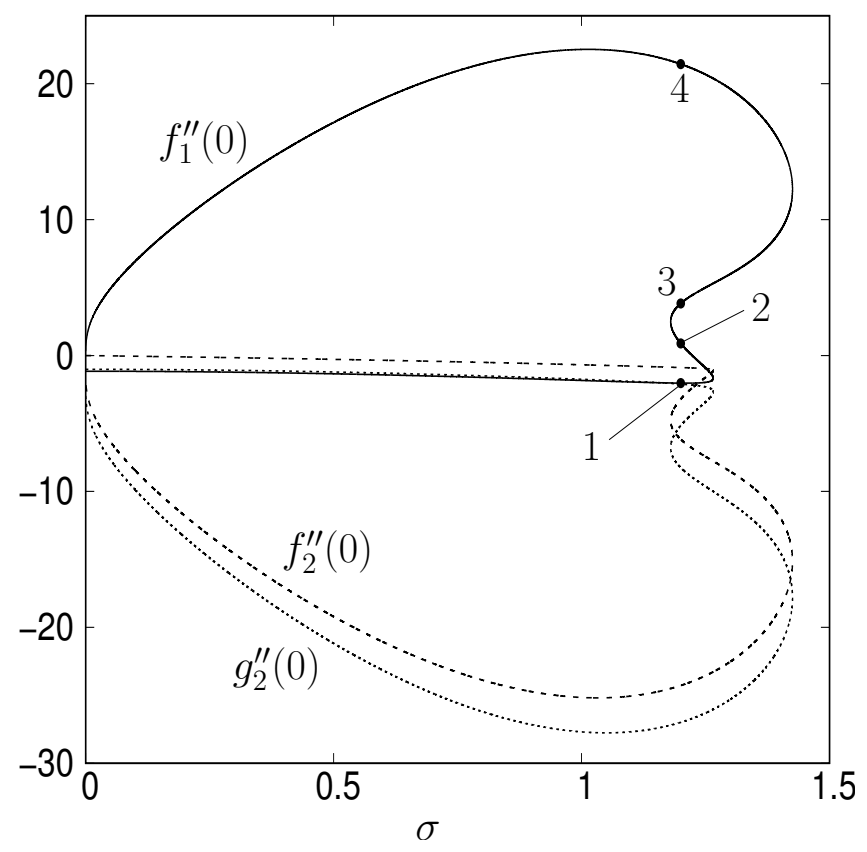

Figure 5a. Plate stress parameters $f_{1}^{\prime \prime}(0), f_{2}^{\prime \prime}(0) g_{2}^{\prime \prime}(0)$ as a function of $\sigma$ for $\lambda=0.9$. The three turning point values $\sigma_{\max }$ for the results shown are 1.180, 1.265 and 1.425 . The velocity profiles for solutions with $f_{1}^{\prime \prime}(0)$ values numbered 1-4 are plotted in figures $4(\mathrm{~b}-\mathrm{e})$.

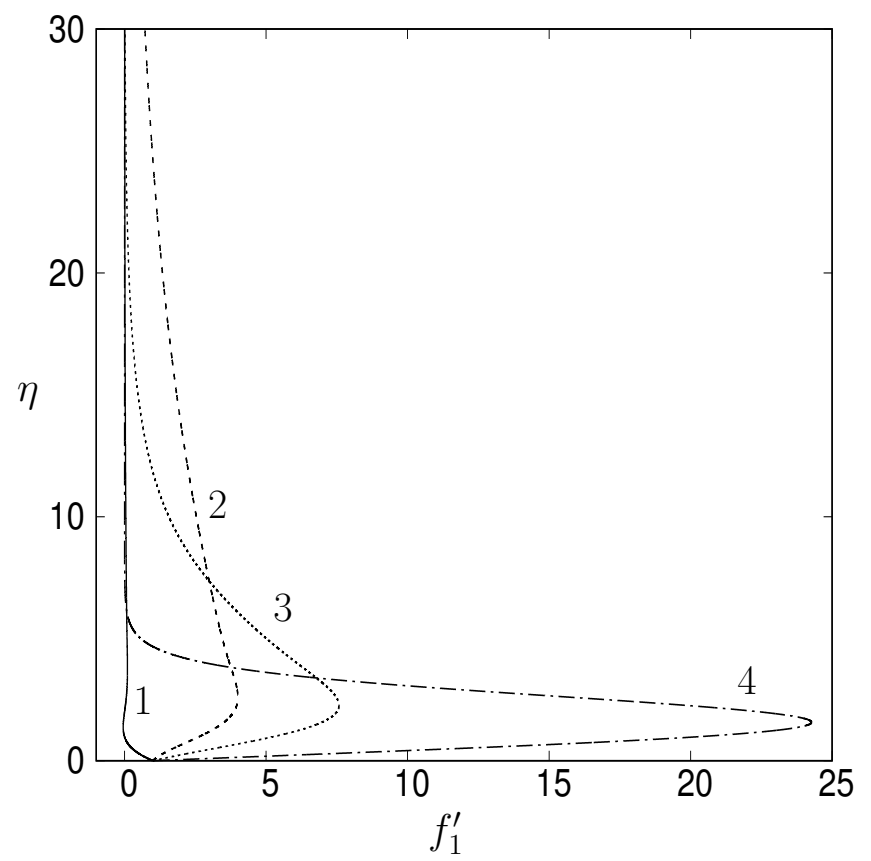

Figure 5b. Velocity profile $f_{1}^{\prime}(\eta)$ for $\sigma=1.2$ and $\lambda=0.9$. The results numbered 1-4 correspond to the solutions numbered in figure $5 \mathrm{a}$. 


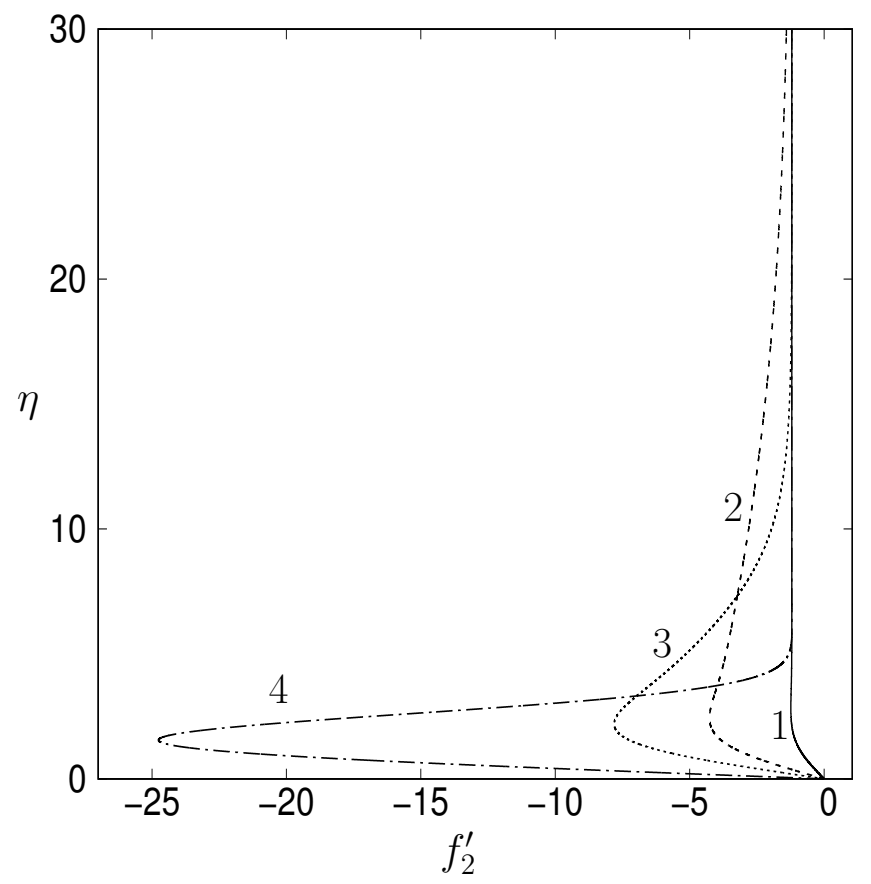

Figure 5c. Velocity profile $f_{2}^{\prime}(\eta)$ for $\sigma=1.2$ and $\lambda=0.9$. The results numbered 1-4 correspond to the solutions numbered in figure $5 \mathrm{a}$.

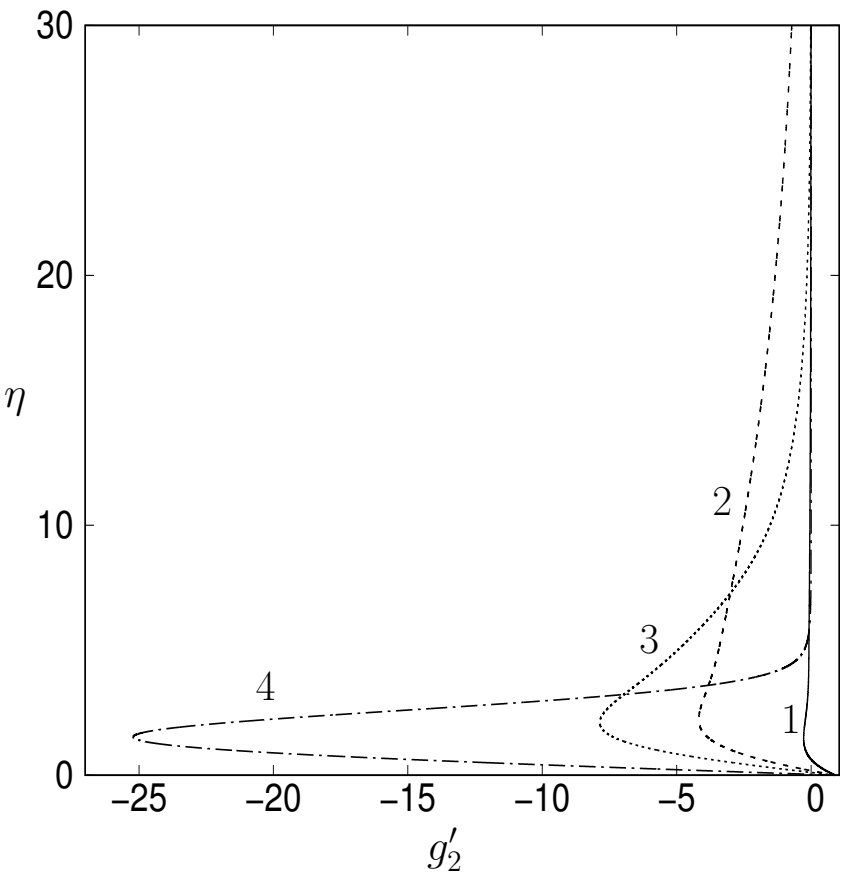

Figure 5d. Velocity profile $g_{2}^{\prime}(\eta)$ for $\sigma=1.2$ and $\lambda=0.9$. The results numbered 1-4 correspond to the solutions numbered in figure $5 \mathrm{a}$. 


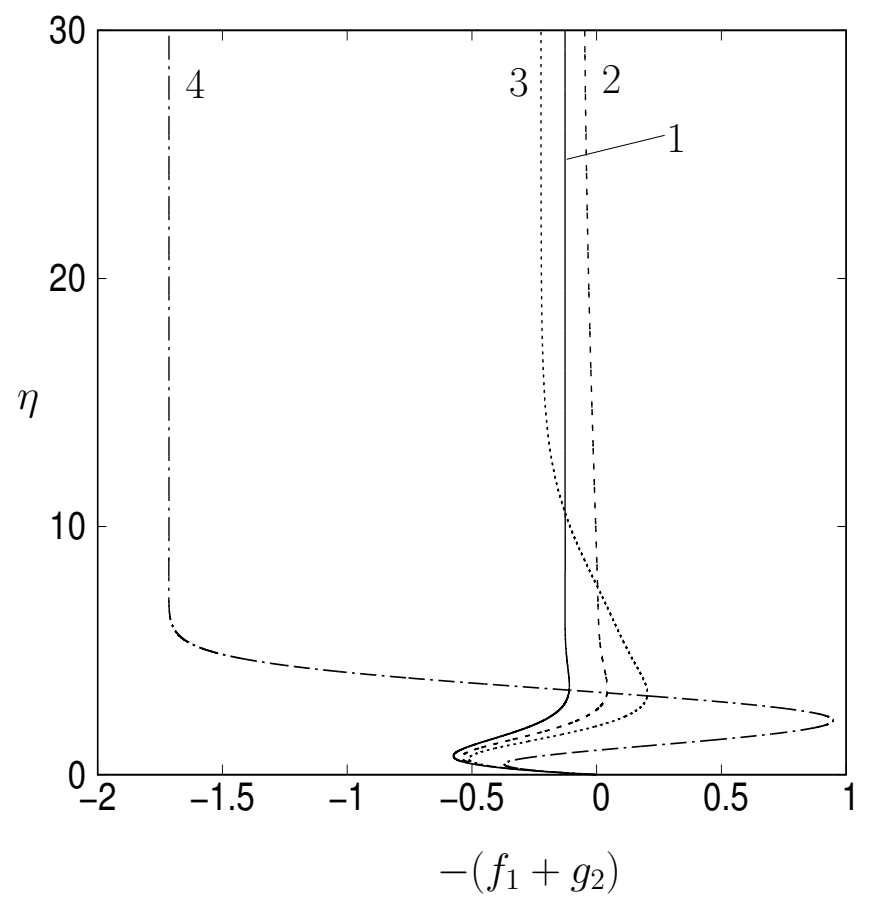

Figure 5e. Velocity profile $-\left(f_{1}+g_{2}\right)(\eta)$ for $\sigma=1.2$ and $\lambda=0.9$. The results numbered 1-4 correspond to the solutions numbered in figure $5 \mathrm{a}$.

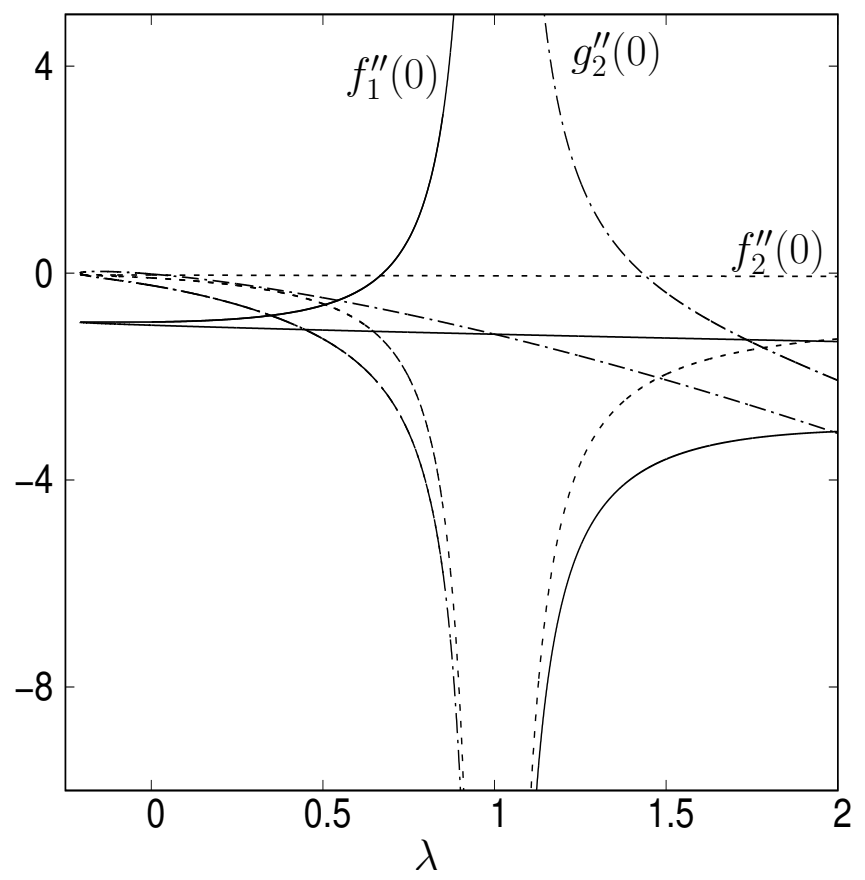

Figure 6a. Plate stress parameters $f_{1}^{\prime \prime}(0), f_{2}^{\prime \prime}(0) g_{2}^{\prime \prime}(0)$ as a function of $\lambda$ for $\sigma=0.1$. The minimum value $\lambda_{\min }$ in this case is -0.208 . 


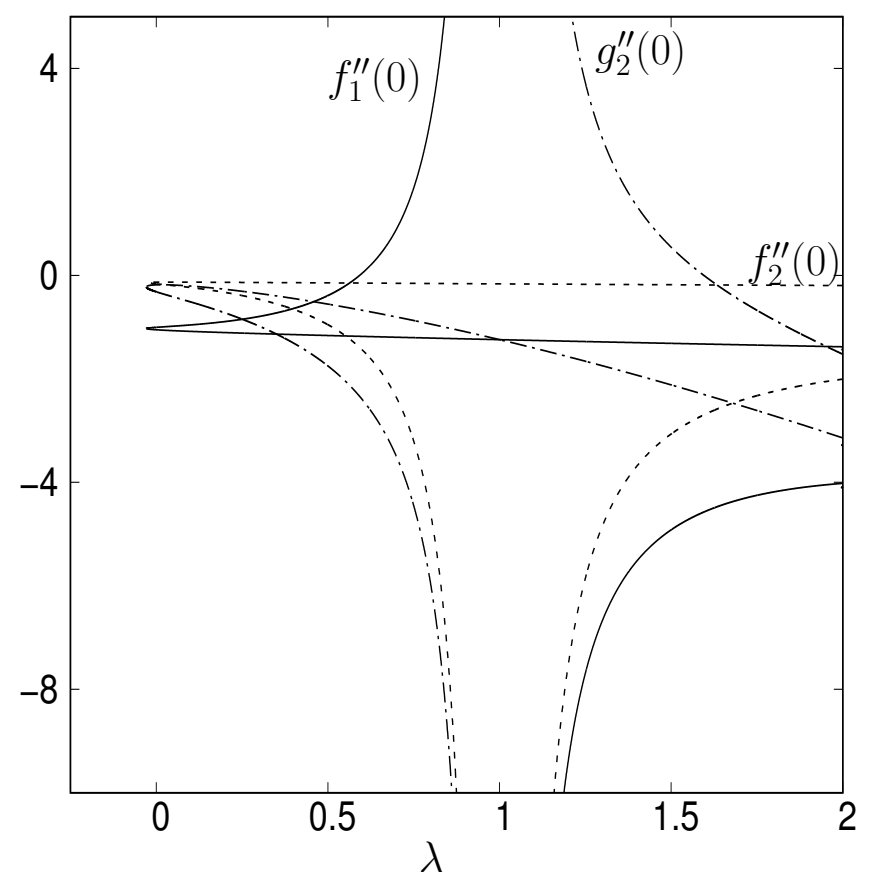

Figure 6b. Plate stress parameters $f_{1}^{\prime \prime}(0), f_{2}^{\prime \prime}(0) g_{2}^{\prime \prime}(0)$ as a function of $\lambda$ for $\sigma=0.3$. The minimum value $\lambda_{\min }$ in this case is -0.029 .

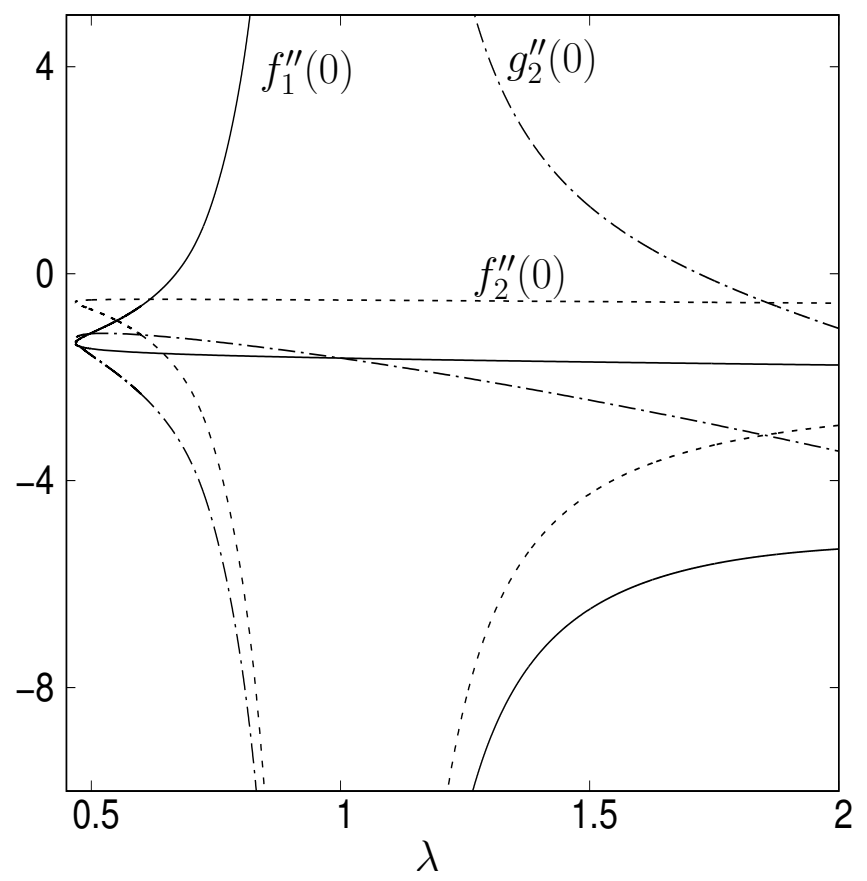

Figure 6c. Plate stress parameters $f_{1}^{\prime \prime}(0), f_{2}^{\prime \prime}(0) g_{2}^{\prime \prime}(0)$ as a function of $\lambda$ for $\sigma=0.8$. The minimum value $\lambda_{\min }$ in this case is 0.468 . 


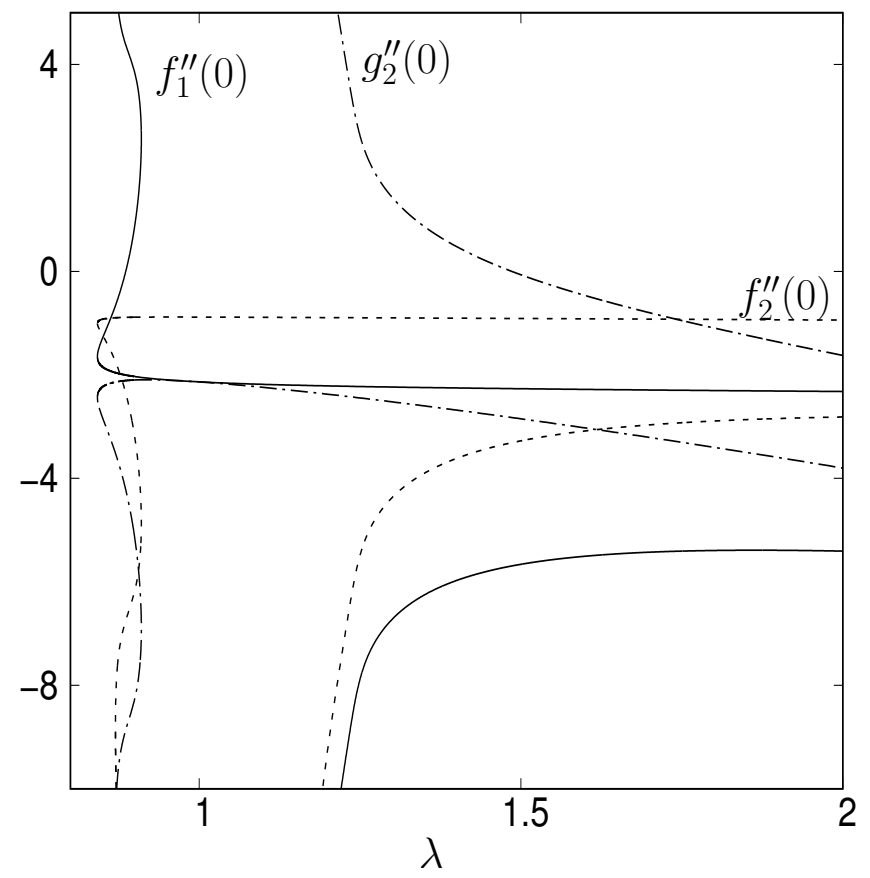

Figure 6d. Plate stress parameters $f_{1}^{\prime \prime}(0), f_{2}^{\prime \prime}(0) g_{2}^{\prime \prime}(0)$ as a function of $\lambda$ for $\sigma=1.2$. The minimum value $\lambda_{\min }$ in this case is 0.842 .

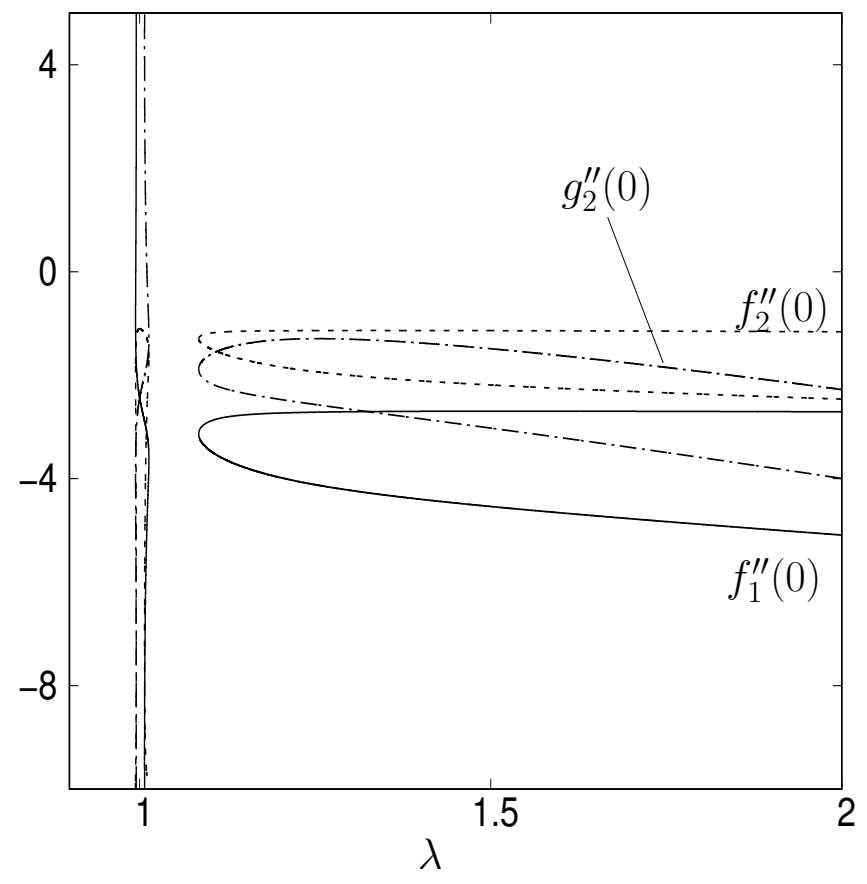

Figure 6e. Plate stress parameters $f_{1}^{\prime \prime}(0), f_{2}^{\prime \prime}(0) g_{2}^{\prime \prime}(0)$ as a function of $\lambda$ for $\sigma=1.4$. The minimum value $\lambda_{\min }$ on the two branches emanating from $\lambda=\infty$ is 1.084 . 


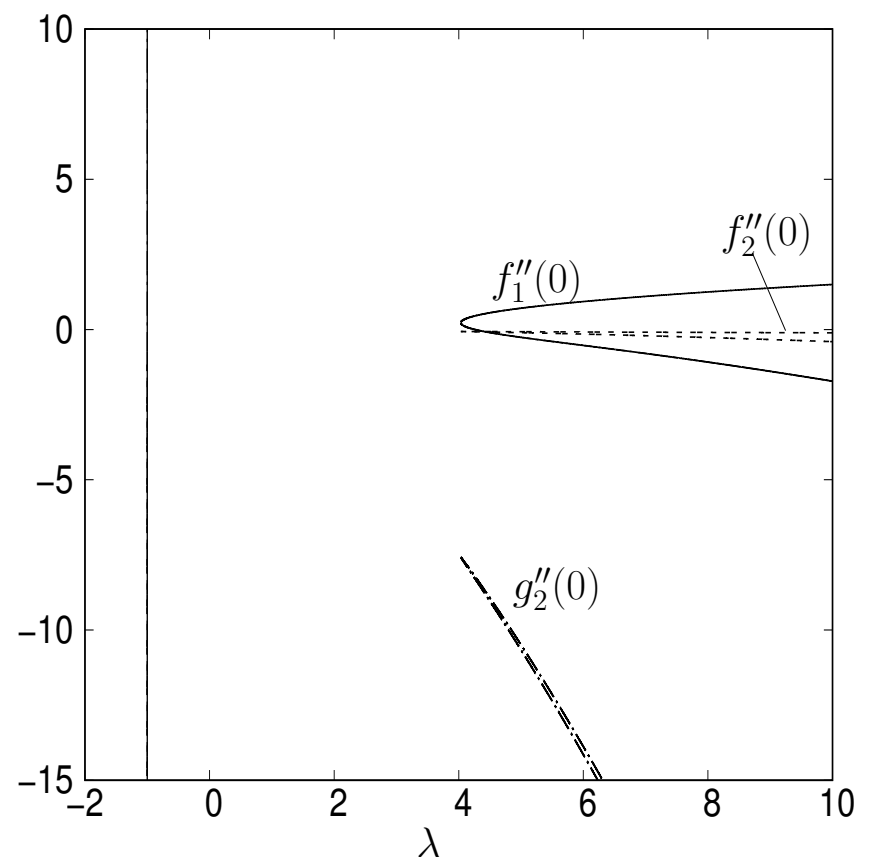

Figure 7a. Plate stress parameters $f_{1}^{\prime \prime}(0), f_{2}^{\prime \prime}(0) g_{2}^{\prime \prime}(0)$ as a function of $\lambda$ for $\sigma=$ 0.1 with $f_{1}^{\prime}(0)=-1$. The minimum value $\lambda_{\text {min }}$ on the two branches emanating from $\lambda=\infty$ is 4.033 .

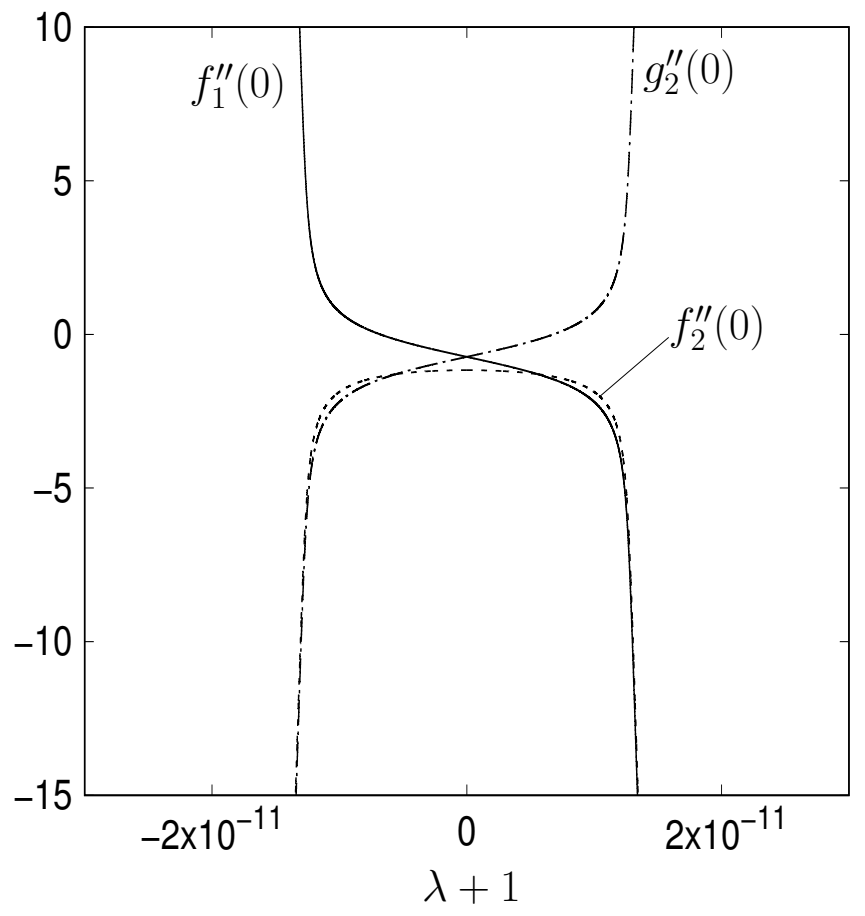

Figure $7 \mathrm{~b}$. Blow up of figure 7 a close to $\lambda=-1$. 


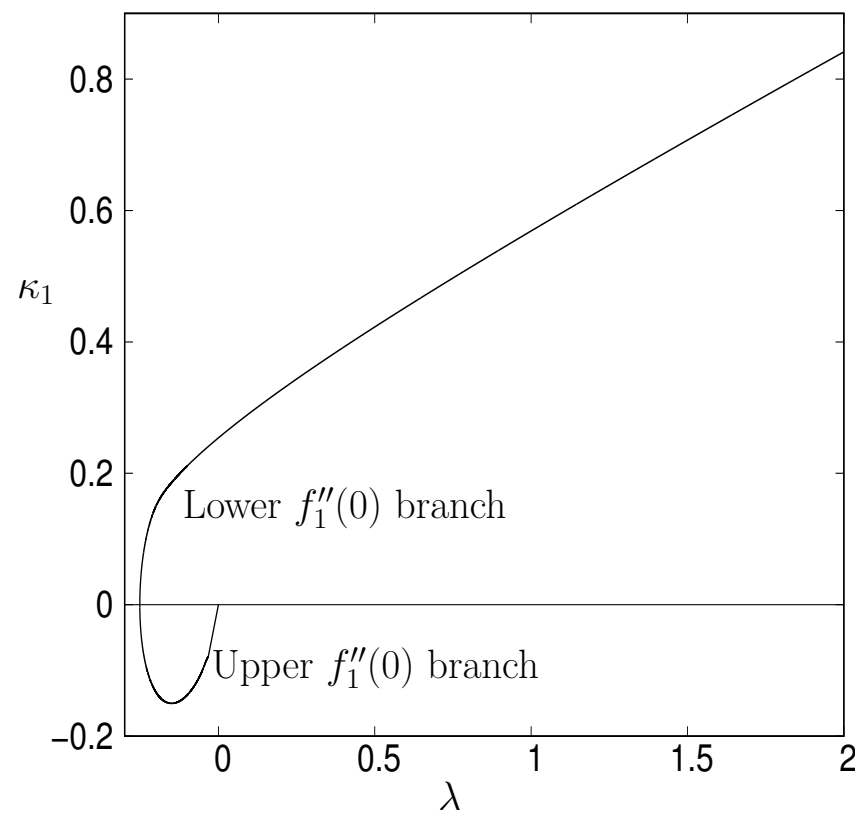

Figure 8a. Lowest eigenvalue $\kappa_{1}(\lambda)$ for the case $\sigma=0$ from figure 2 a. For $\lambda<0$ the lower $f_{1}^{\prime \prime}(0)$ branch results from figure $2 \mathrm{a}$ are stable, and the corresponding upper branch results are unstable.

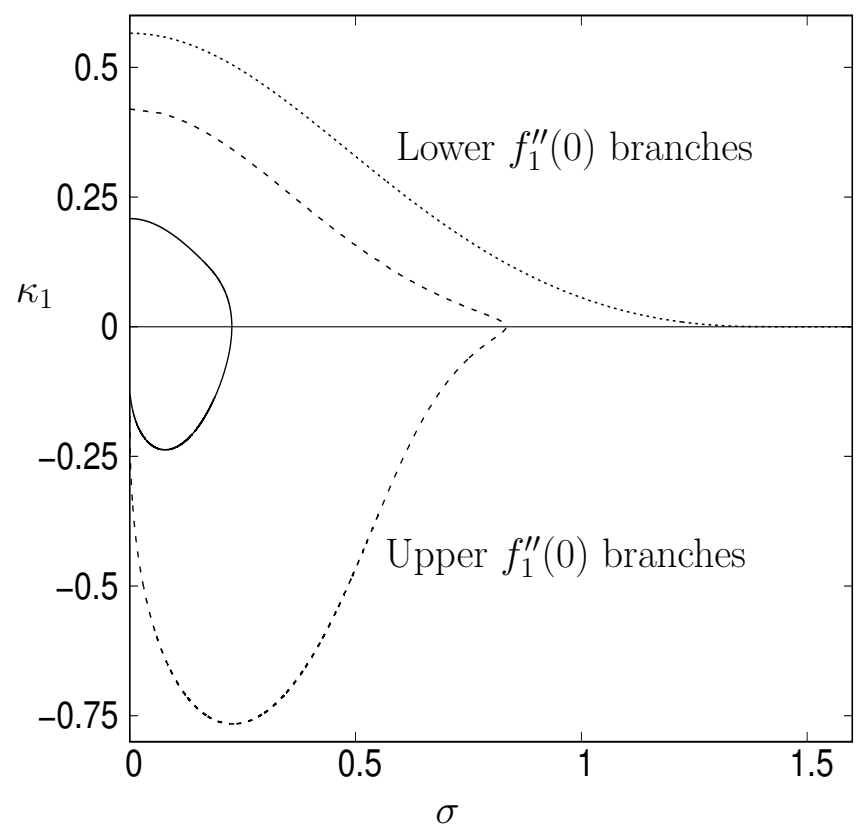

Figure 8b. Lowest eigenvalue $\kappa_{1}(\sigma)$ for the $\lambda=-0.1$ (solid line), $\lambda=0.5$ (dashed line) and $\lambda=1$ (short-dashed line) from figures $3(\mathrm{a}-\mathrm{c})$. For $\lambda=-0.1$ and 0.5 the lower $f_{1}^{\prime \prime}(0)$ branch results from figure $3 \mathrm{a}$ are stable, and the corresponding upper branch results are unstable, while for $\lambda=1$ (which only has a lower $f_{1}^{\prime \prime}(0)$ branch) results are stable for all $\sigma$. 